CALT-68-2860

UCI-TR-2013-18

\title{
Self-Interacting Dark Matter from a Non-Abelian Hidden Sector
}

\author{
Kimberly K. Boddy, ${ }^{1}$ Jonathan L. Feng, ${ }^{2}$ Manoj Kaplinghat, ${ }^{2}$ and Tim M. P. Tait ${ }^{2}$ \\ ${ }^{1}$ California Institute of Technology, Pasadena, California 91125, USA \\ ${ }^{2}$ Department of Physics and Astronomy, \\ University of California, Irvine, California 92697, USA
}

\begin{abstract}
There is strong evidence in favor of the idea that dark matter is self-interacting, with cross section-tomass ratio $\sigma / m \sim 1 \mathrm{~cm}^{2} / \mathrm{g} \sim 1$ barn $/ \mathrm{GeV}$. We show that viable models of dark matter with this large cross section are straightforwardly realized with non-Abelian hidden sectors. In the simplest of such models, the hidden sector is a pure gauge theory, and the dark matter is hidden glueballs with mass around $100 \mathrm{MeV}$. Alternatively, the hidden sector may be a supersymmetric pure gauge theory with a $\sim 10 \mathrm{TeV}$ gluino thermal relic. In this case, the dark matter is largely composed of glueballinos that strongly self-interact through the exchange of light glueballs. We present a unified framework that realizes both of these possibilities in anomaly-mediated supersymmetry breaking, where, depending on a few model parameters, the dark matter is either hidden glueballinos, hidden glueballs, or a mixture of the two. These models provide simple examples of multi-component dark matter, have interesting implications for particle physics and cosmology, and include cases where a sub-dominant component of dark matter may be extremely strongly self-interacting, with interesting astrophysical consequences.
\end{abstract}

PACS numbers: 95.35.+d, 12.60.Jv 


\section{INTRODUCTION}

The standard model of cosmology is the $\Lambda \mathrm{CDM}$ model, in which the contents of the Universe are dominated by vacuum energy $\Lambda$ and collisionless cold dark matter (CDM). The success of the $\Lambda \mathrm{CDM}$ model is based on its well-established record of success in describing the features of the large-scale structure observed in the Universe.

On smaller scales, however, the picture is much less clear. $N$-body simulations of collisionless CDM appear to disagree with observations on small scales, motivating dark matter properties that differ significantly from the standard paradigm. In particular, if dark matter is self-interacting (able to scatter elastically with itself), simulations show that the core sizes and central densities of dwarf spheroidal galaxies, low-surface-brightness spirals, and galaxy clusters can all be brought in line with observations [1-4]. This modification to $\Lambda \mathrm{CDM}$, sometimes called $\Lambda$ SIDM, is consistent with constraints from the Bullet Cluster, measurements of dark matter halo shapes, and subhalo survival requirements. To make the simulations and observations consistent, the ratio of the dark matter's self-interaction cross section to its mass should be in the range $\sigma / m \sim 0.1-10 \mathrm{~cm}^{2} / \mathrm{g}$. The requirement of such strong self-interactions eliminates from consideration all of the most studied dark matter candidates, including weakly-interacting massive particles (WIMPs), axions, and sterile neutrinos.

At the same time, such large cross sections are on par with nuclear-scale cross sections $\left(1 \mathrm{~cm}^{2} / \mathrm{g} \simeq 1.78 \mathrm{barn} / \mathrm{GeV}\right)$. The possibility that dark matter has color and interacts through the strong interactions of the standard model (SM) is highly constrained, for example, by searches for anomalous isotopes in sea water [5-7]. However, dark matter may self-interact through nonAbelian forces (such as a dark analogue of QCD) in a hidden sector. As we will show below, this is straightforwardly realized in even the simplest such hidden sectors, with $\mathrm{SU}(N)$ gauge symmetry and no additional matter content. For confinement scales $\Lambda \sim 100 \mathrm{MeV}$, the hidden gluons confine to form glueballs, and the resulting glueball dark matter has the required self-interactions. For hidden sectors that are roughly the same temperature as the visible sector, the glueball relic density is generically too large, but the desired relic density may be obtained by adjusting the relative temperatures of the hidden sector and visible sector, as we discuss below.

This hidden glueball scenario for self-interacting dark matter is remarkably simple, but it is decoupled from the visible sector, both in the technical sense and in the sense that it is not motivated by any of the well-known problems of the SM. In addition, the correct relic density is arranged by tuning a free parameter, the ratio of hidden to visible sector temperatures, and so the scenario cannot be claimed to naturally produce the right thermal relic density, as in the case of WIMPs. At first sight, it might appear to be difficult to enhance the model to accommodate all of these desirable features, especially since the WIMP miracle requires weak-scale annihilation cross sections, whereas the required self-interactions naturally suggest strong interactions.

In fact, however, we will show that all of these features are present in a supersymmetric version of the hidden glueball scenario, in which the hidden sector is a supersymmetric pure gauge theory. In this model, the dark matter is a $\sim 10 \mathrm{TeV}$ hidden gluino, which freezes out in the early Universe when the temperature is high. At freezeout, the theory is weakly coupled, but as the Universe cools and expands, the theory confines, forming hidden glueballinos and glueballs. The glueballinos strongly interact via exchange of the hidden glueballs with the required selfinteraction cross section. This scenario is straightforwardly accommodated in anomaly-mediated supersymmetry breaking (AMSB) scenarios [8,9], which provides a connection to the problems of the SM and also allows the glueballinos to naturally inherit the correct relic density through the WIMPless miracle [10, 11], a possibility discussed previously in Refs. [12-14]. For related work on strongly-interacting hidden sectors and dark matter, see Refs. [15-22].

Of course, the supersymmetric models also contain glueballs, which, as in the non- 


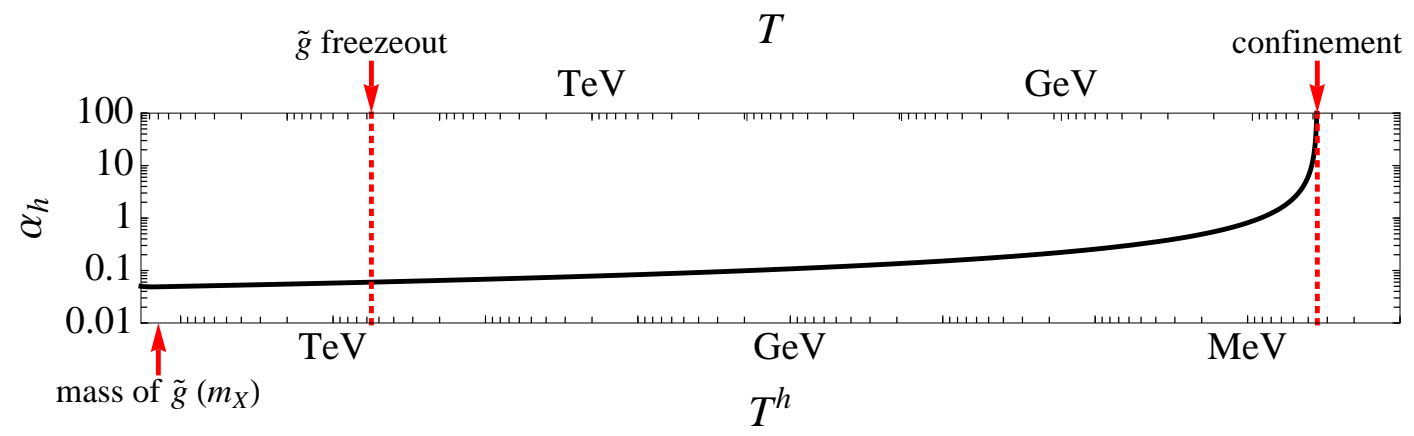

FIG. 1: Example timeline of events in the supersymmetric pure $\mathrm{SU}(N)$ theory without connectors, in terms of the hidden- and visible-sector temperatures $T^{h}$ and $T$. The hidden sector coupling $\alpha_{h}$ is shown as a function of these temperatures. It is weak at gluino freezeout but grows as the temperature drops, leading to confinement and the formation of glueballino and glueball dark matter at a temperature $\sim \Lambda$. The scenario is described in detail in Sec. VI, and the chosen parameters are represented by the yellow dot in Fig. 5.

supersymmetric case, may be dark matter. As we will see, in different regions of the AMSB parameter space, the dark matter may be dominantly glueballinos, dominantly glueballs, or a mixture of the two. For the case where the dark matter is dominantly glueballinos, we detail two possibilities. In the first case, the hidden sector is coupled to the visible sector only indirectly through the supersymmetry breaking mechanism. Since this coupling is extremely weak, the sectors can have different temperatures, and the glueball relic density may be very small for cold hidden sectors. An example cosmological timeline of events in this case is given in Fig. 1.

In the second case, the hidden sector is coupled to the visible sector through connector fields. The visible and hidden sectors, therefore, have the same temperature at early times, leading, a priori, to too-large glueball relic densities. Decays of glueballs are in conflict with big bang nucleosynthesis $(\mathrm{BBN})$ and other astrophysical and particle constraints. Instead, we rely on a novel non-thermal process in the early Universe to deplete the gluon density, thereby suppressing the glueball density after confinement. In this case, the gluons annihilate into singlet right-handed neutrinos with $\sim 1 \mathrm{GeV}$ mass, and we reduce the hidden gluon density by forcing the right-handed neutrinos to decay into SM particles more quickly than they can annihilate back into hidden gluons. A representative timeline for this case is shown in Fig. 2.

This work is organized as follows. In Sec. II, we review the astrophysical evidence for selfinteracting dark matter. In Sec. III, we begin with the simplest possible case: non-supersymmetric pure gauge hidden sectors and glueball dark matter. We discuss glueball self-interactions and relic densities and determine the preferred parameters for this simple model. We then move to supersymmetric models with pure gauge hidden sectors and glueballino dark matter. In Sec. IV, we review the calculation of the glueballino self-interaction cross section, and in Sec. V, we discuss the glueballino relic density and the realization of the WIMPless miracle in the AMSB framework. Finally, with this groundwork, we present full AMSB models of glueballino/glueball dark matter without and with connectors in Secs. VI and VII, respectively. We conclude in Sec. VIII.

Last, a note on naming conventions. In the rest of this work, we follow the literature: glueballinos denote gluino-gluon bound states, while gluinoballs denote gluino-gluino bound states. In addition, unless otherwise stated, "gluon," "gluino," "glueball," and "glueballino" refer to hidden sector particles and are denoted by $g, \tilde{g}$, gb, and gbino, respectively. 


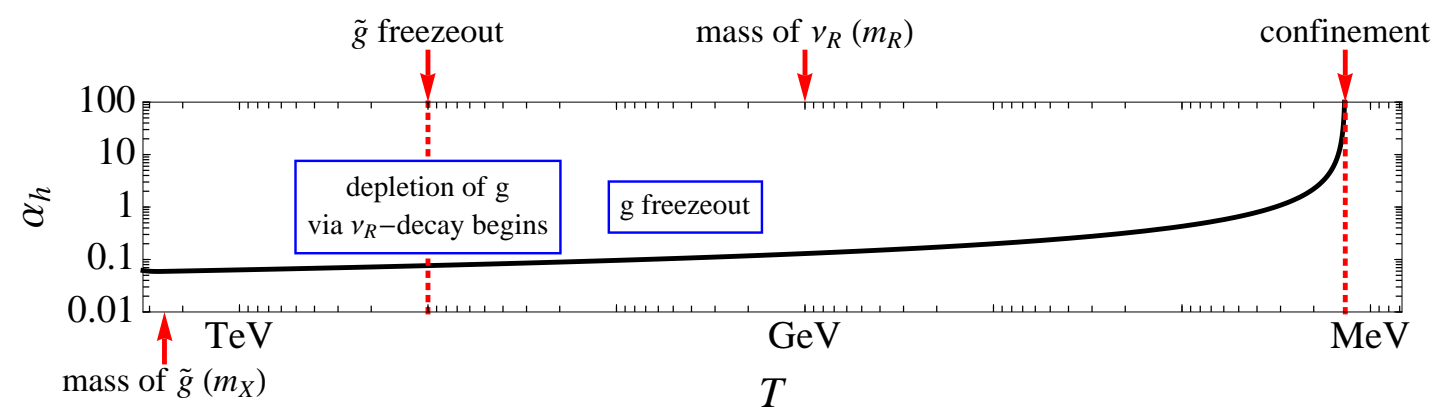

FIG. 2: As in Fig. 1, but for supersymmetric pure SU $(N)$ theory with connectors. Since the hidden and visible sectors communicate efficiently in the early Universe, they share a temperature $T$. The gluon population is depleted through their decays to the $\nu_{R}$ in the visible sector, and the resulting scenario has pure glueballino dark matter. The scenario is described in detail in Sec. VII, and the chosen parameters are represented by the yellow dot in Fig. 8 .

\section{ASTROPHYSICAL EVIDENCE FOR SELF-INTERACTING DARK MATTER}

The $\Lambda$ CDM model is quite successful in describing large-scale structure. The predictions of the standard six-parameter $\Lambda \mathrm{CDM}$ cosmology match remarkably well to the latest measurements of the cosmic microwave background (CMB) by WMAP [23] and Planck [24] at large multipoles of the power spectrum. Additionally, CDM fits the dark matter power spectrum very well [25], using observations of luminous red galaxy clustering in the Sloan Digital Sky Survey [26].

Despite these agreements on large scales, observations of small-scale structures indicate that $\mathrm{CDM}$ is insufficient. Challenges to the $\Lambda \mathrm{CDM}$ paradigm arise largely from tensions between observation and cosmological simulations. Simulations of CDM create dark matter halos with density profiles that have steep, inverse-power law behaviors (cusps) towards the center of the halo [27-31]. Conversely, observations show that low-surface-brightness spiral galaxies (LSBs) [32-39], satellite dwarf galaxies [38, 40], and galaxy clusters [41-46] exhibit constant-density cores. In addition to the core-cusp discrepancy [47], the simulated central densities of halos are too high. By matching the luminosity function of the Milky Way to the Aquarius simulations [48], the brightest subhalos in the Milky Way are a factor of 5 less massive than predicted [49, 50]. If $\Lambda$ CDM is correct, we are left to explain this "too-big-to-fail" problem in which the largest subhalos of the Milky Way do not luminesce; otherwise, some additional physics is needed in simulations to decrease the central densities of these overly-massive halos.

To address these concerns with $\Lambda \mathrm{CDM}$, there are a few generic possibilities to consider [51]: adding feedback from baryons in simulations [52-54], warm dark matter (WDM) [55-57], and selfinteracting dark matter [58-60]. Feedback exists and should be included in simulations, but there may not be enough energy to eject a sufficient amount of mass from the halo center to solve the too-big-to-fail problem [50]. WDM tends to be too efficient in wiping out structure, leaving too few subhalos in the Milky Way [61]. Additionally, lower bounds on WDM masses from Lyman- $\alpha$ forest measurements constrain the ability of WDM to solve the core-cusp problem over the full range of astrophysical scales needed $[62,63]$. Even with its mass unconstrained, WDM still leaves dwarf halos cuspy, though it does lower the central densities [64].

On the other hand, self-interacting dark matter can soften halo cores and lower central densities, while preserving large-scale structure [58] and satisfying bounds of $\sigma / m \lesssim 1 \mathrm{~cm}^{2} / \mathrm{g}$ from the Bullet Cluster [65]. Indeed, simulations with constant dark matter cross section-to-mass ratios in the range 
$0.1-1 \mathrm{~cm}^{2} / \mathrm{g}$ show that self-interactions can bring theory in line with observations of both halo profiles and shapes $[1,2]$. Velocity-dependent self-interactions widen this range to $0.1-10 \mathrm{~cm}^{2} / \mathrm{g}$ and can also soften cores and reduce the density of the brightest satellites to solve the too-big-to-fail problem $[3,4]$.

With these results from simulation, dark matter with self-interactions is a strong contender within particle physics to be a solution to the small-scale formation woes in astrophysics. From a particle physics perspective, we will see that self-scattering is a quite reasonable and perhaps even generic property for dark matter to possess.

\section{GLUEBALL DARK MATTER}

The simplest construction resulting in dark matter that is a composite of a strongly interacting hidden sector is a pure Yang-Mills gauge theory. At large energy scales, the theory consists of a weakly coupled set of massless gluons whose couplings are described by the gauge coupling. The theory is expected to confine at low energies at a scale $\Lambda$, where the gauge coupling becomes strong enough that perturbation theory breaks down [66-72]. At this point it develops a mass gap, and the low energy physics is described by a set of glueball states $(\mathrm{gb})$ whose masses are characterized by $\Lambda$ through dimensional transmutation.

At very low energies $\ll \Lambda$, the physics is described by an effective field theory composed of the the low-lying glueball states. In the absence of any coupling to the SM, the lightest of these states will be effectively stable. ${ }^{1}$ The detailed mass spectrum (and spins) of these states depends on the underlying choice of theory and is further clouded by strong coupling, which leaves results based on perturbation theory suspect. Generically, one expects the glueball spectrum to have a lowest lying element whose mass is $\mathcal{O}(\Lambda)$, which, following the guidance of QCD, we take to be a $J^{C P}=0^{++}$ state $[73,74]$. There will also be a collection of excited states with various spins and whose mass splittings are roughly multiples of $\Lambda$.

\section{A. Glueball Self-Interactions}

The various glueball states will interact with one another as a residual of the strong dynamics that binds them. Dimensional analysis dictates that the interactions among them will be proportional to $\Lambda$ to an appropriate power, with dimensionless coefficients characterized by naïve dimensional analysis (NDA) $[75,76]$. For example, a description of a scalar glueball state $\phi_{0}$ would look like,

$$
\mathcal{L}_{\mathrm{gb}}=\frac{1}{2} \partial_{\mu} \phi_{0} \partial^{\mu} \phi_{0}-\frac{1}{2} m^{2} \phi_{0}^{2}+\frac{A}{3 !} \phi_{0}^{3}+\frac{\lambda}{4 !} \phi_{0}^{4}+\ldots
$$

where NDA would suggest that for the lowest lying state $m \simeq \Lambda$, and $A \simeq(4 \pi) \Lambda, \lambda \simeq(4 \pi)^{2}$, and the $+\ldots$ indicates interaction terms in the form of higher dimensional operators that are suppressed by powers of $\Lambda$. Interactions involving the various glueball excited states can be formulated in a similar way.

For energies $\ll \Lambda$, the physics should be well-described by an effective field theory composed of the lightest glueball. At kinetic energies of order $\Lambda$, more of the lowest lying states become accessible and need to be included in the effective theory. At energies $\gg \Lambda$, the physics is described by the

\footnotetext{
${ }^{1}$ Note that gravitational interactions will mediate very suppressed decays to light SM particles, but these are irrelevant for $\Lambda \ll M_{\mathrm{Pl}}$.
} 
interactions of the gluons together with the structure functions that describe their distribution inside of the glueballs.

Although it is clear that glueballs are strongly self-interacting, it is very difficult to make precise predictions for the scattering rate, given our general ignorance concerning strongly coupled theories. The expected cross section will be characterized by the confinement scale and strong coupling,

$$
\sigma(\mathrm{gb} \mathrm{gb} \rightarrow \mathrm{gb} \mathrm{gb}) \simeq \frac{(4 \pi)^{2}}{\Lambda^{2}},
$$

which can also be understood from the geometric size of the glueballs, whose radius is $\sim 1 / \Lambda$.

\section{B. Glueball Relic Density}

If the glueballs are stable on the scale of the age of the Universe, they will contribute to the total observed dark matter relic density. At early times, when their temperature is $T_{h} \gg \Lambda$, the hidden sector is represented by a plasma of gluons whose comoving relativistic number density is given by

$$
Y_{\infty}=\frac{n_{g}}{s}=\left.\frac{\left[\zeta(3) / \pi^{2}\right] g_{\mathrm{eff}} T^{h^{3}}}{\left(2 \pi^{2} / 45\right) g_{* S} T^{3}}\right|_{t_{f}}=\frac{45 \zeta(3)}{2 \pi^{4}} \xi_{f}^{3} \frac{g_{\mathrm{eff}}}{g_{* S}\left(t_{f}\right)}
$$

where $s$ is the entropy in the visible sector, $\xi_{f} \equiv T^{h} / T$ is the ratio of temperatures in the hidden and visible sectors, $\zeta(3) \approx 1.202$ is the zeta function, and $g_{\mathrm{eff}}=2\left(N^{2}-1\right)$ for an $\mathrm{SU}(N)$ gauge theory. We use an early time $t_{f}$ (which we will identify with the time of gluino freezeout in the supersymmetric models discussed below) as a reference point. The quantity $Y_{\infty}$ remains constant as the Universe expands.

As the hidden sector temperature $T^{h}$ cools below the critical temperature $T^{c} \sim \Lambda$ [77], there is a transition to the confined phase, and the energy density of the gluon plasma is converted into glueballs. The result is that after confinement the Universe is filled with nonrelativistic glueballs whose comoving number density is the same as that of the gluons up to factors of $\mathcal{O}(1)$. Consequently, today the glueballs are nonrelativistic with a relic density

$$
\Omega_{\mathrm{gb}} \sim \frac{Y_{\infty} s_{0} \Lambda}{\rho_{c 0}} .
$$

\section{Viable Glueball Parameters}

Glueball dark matter is thus primarily characterized by two quantities: the confinement scale $\Lambda$, which simultaneously controls the dark matter mass and its self-interaction cross section, and $\xi_{\Lambda}$, the ratio of temperatures of the hidden and visible sectors at the time of confinement. Also relevant are the number of gluon degrees of freedom; for an $\mathrm{SU}(N)$ gauge theory this is specified by $N$ through $g_{\text {eff }}=2\left(N^{2}-1\right)$. In Fig. 3, we show the parameter space in the $\left(\xi_{\Lambda}, \Lambda\right)$ plane. The scattering cross section is independent of $\xi_{\Lambda}$, which together with the choice of $N$ controls the relic density of glueballs. The scattering cross sections of interest suggest $\Lambda \sim 100 \mathrm{MeV}$, amusingly close to $\Lambda_{\mathrm{QCD}} \approx 300 \mathrm{MeV}$. Note that since the cross section is constant, the acceptable upper limit from simulations is $1 \mathrm{~cm}^{2} / \mathrm{g}$, in particular, to stay within cluster constraints. This limit will increase to $10 \mathrm{~cm}^{2} / \mathrm{g}$ for velocity-dependent cross sections, which we begin discussing in Sec. IV. The relic density requires the hidden sector temperature to be a few orders of magnitude below the visible temperature at the time of confinement. 


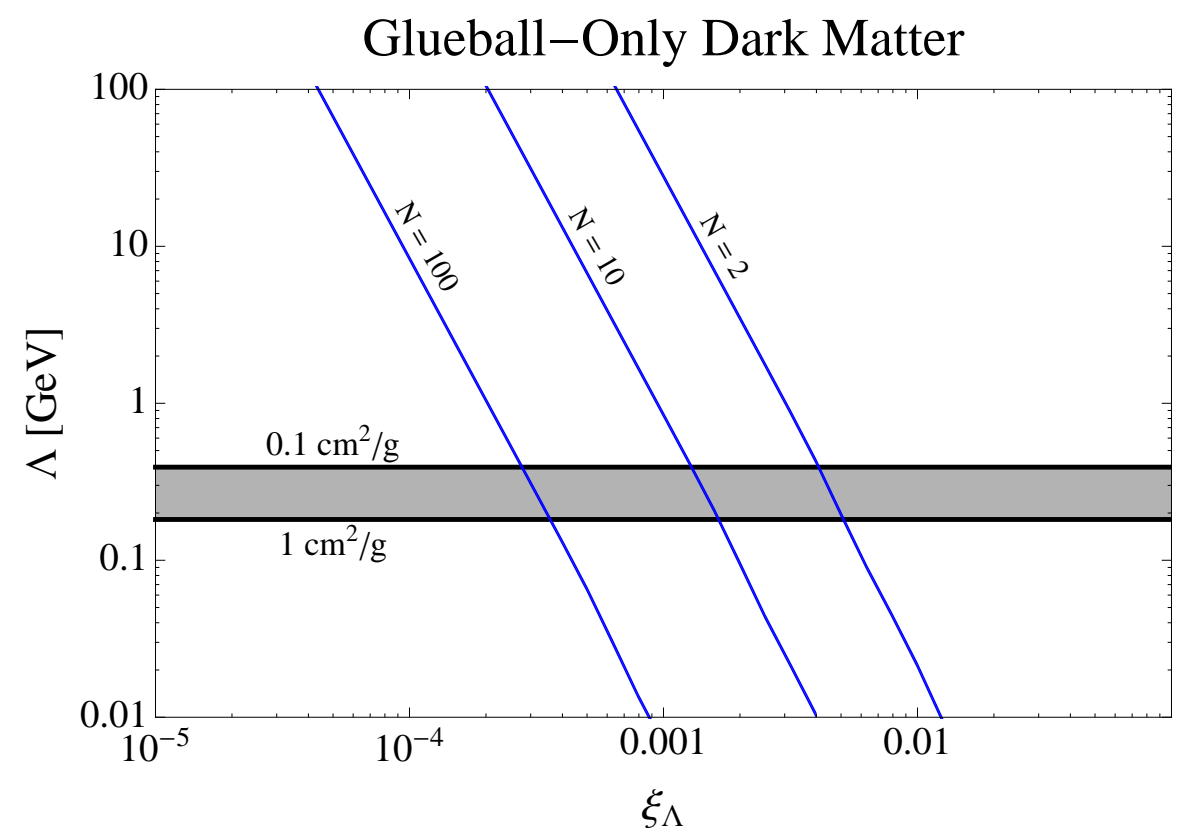

FIG. 3: Glueball dark matter in the case of a non-supersymmetric pure gauge $\operatorname{SU}(N)$ hidden sector. The self-interaction cross section and relic density are given in the $\left(\xi_{\Lambda}, \Lambda\right)$ plane, where $\Lambda$ is the confinement scale in the hidden sector, and $\xi_{\Lambda} \equiv T^{h} / T$ is the ratio of hidden to visible sector temperatures at the time that $T^{h}=\Lambda$. The self-interaction cross section is in the range $\left\langle\sigma_{T}\right\rangle / m_{X}=0.1-1 \mathrm{~cm}^{2} / \mathrm{g}$ in the shaded region. The glueball relic density is $\Omega_{\mathrm{gb}}=\Omega_{\mathrm{DM}} \simeq 0.23$ on the diagonal contours for the number of colors $N$ indicated.

\section{GLUEBALLINO SELF-INTERACTIONS}

The simplest extension to the pure gauge hidden sector discussed in Sec. III is to add a massive (mass $m_{X} \gg \Lambda$ ) gauge adjoint Majorana fermion to the theory, resulting in a spectrum with two types of composites: the bosonic glueballs of mass $\sim \Lambda$ and the fermionic states with masses $\sim m_{X}$ [78-81]. Each sector contains excited states whose mass splittings are again characterized by $\Lambda$. In the absence of further ingredients, the massive fermionic states are stable because of Lorentz invariance, and this construction allows one to realize a situation where the dark matter is (mostly) composed of the heavy composite fermions that self-interact via exchange of the much lighter glueballs, naturally realizing two widely separated energy scales. This dark sector is identical to a softly broken $N=1$ supersymmetric gauge theory and can be considered the supersymmetric version of the model of Sec. III. In that language, the composite fermions are glueballino states.

The self-interactions of glueballinos are dominated by the exchange of glueballs. At low energies, when the kinetic energy available is $\lesssim \Lambda$, the scattering will be elastic. If there is sufficient kinetic energy,

$$
\frac{1}{2} m_{X} v^{2} \geq \Lambda
$$

inelastic scattering into excited states and glueball emission becomes possible, leading to novel effects, such as additional rapid halo cooling. The inelastic effects are not modeled in the $\Lambda$ SIDM simulations and so are not well understood. For the remainder of this work, we focus on the elastic scattering regime and comment later in this section on systems where this approximation breaks down.

NDA suggests that the coupling between glueballs and glueballinos is $\alpha \sim 1$. Even for elastic 
scattering, there will be a large number of distinct glueball states, which are capable of mediating self-interactions of the glueballinos, but the dominant contribution arises from the lightest glueball states, which mediate the longest range interactions. Thus, we model the induced potential between two glueballinos as an attractive Yukawa interaction of range $\Lambda$ and strength $\alpha \sim 1$,

$$
V(r)=-\frac{\alpha}{r} \exp (-\Lambda r)
$$

It is common to use the transfer cross section

$$
\sigma_{T}=\int d \Omega(1-\cos \theta) \frac{d \sigma}{d \Omega}
$$

to compare predictions to observation and simulation. We have numerically solved the Schrödinger equation to calculate $\sigma_{T}$, following the methods of Ref. [82]. For the astrophysical systems of interest, to achieve the desired cross sections of $0.1-10 \mathrm{~cm}^{2} / \mathrm{g}$ with $m_{X} \gtrsim \mathrm{TeV}$, the parameters must be in the classical scattering regime, $m_{X} v \gg \Lambda$. Scattering from Yukawa potentials has been studied in this regime in the context of classical, complex plasmas [83-85], and simple analytic fits to numerical results for the transfer cross section have been derived. These plasma physics results may be applied directly to the present dark matter case [86] (in fact, they describe the dark matter model exactly, whereas the Yukawa potentials are only an approximation to screened Coulomb interactions in the plasma physics context), and we have checked that these agree well with our numerical results.

Within a galactic halo or cluster, the dark matter particles have a velocity distribution that we take to be Maxwell-Boltzmann, and so

$$
f\left(v_{i}\right)=\left(\pi v_{0}^{2}\right)^{-3 / 2} e^{-v_{i}^{2} / v_{0}^{2}}
$$

where $v_{0}$ is the mode and $\left\langle v_{i}^{2}\right\rangle=(3 / 2) v_{0}^{2}$ is the square of the three-dimensional velocity dispersion. This distribution is expected for cross sections of $\sigma / m=1.0 \mathrm{~cm}^{2} / \mathrm{g}$ and above [87]; for the slightly lower cross sections that are still of interest to us, the distribution may be modified, but we do not expect this to impact our results significantly. Simulations [1] show that $\left\langle v_{i}^{2}\right\rangle \approx\left(1.2 V_{\max }\right)^{2}$, where $V_{\max }$ is the peak circular velocity of a given system, and thus $v_{0} \approx 0.98 V_{\max }$. The astrophysical systems of interest have values of $V_{\max }$ in the ranges $20-50 \mathrm{~km} / \mathrm{s}$ for dwarfs, $50-130 \mathrm{~km} / \mathrm{s}$ for LSBs, and $700-1000 \mathrm{~km} / \mathrm{s}$ for clusters. We make a simplistic estimate for the dark matter escape velocity, $v_{\text {esc }}^{2}=2 v_{0}^{2}$, so that the largest relative velocity between particles is $2 \sqrt{2} v_{0}$. For two scattering dark matter particles with velocity $\vec{v}_{1}$ and $\vec{v}_{2}$, the velocity-averaged transfer cross section is

$$
\begin{aligned}
\left\langle\sigma_{T}\right\rangle & =\int \frac{d^{3} v_{1} d^{3} v_{2}}{\left(\pi v_{0}^{2}\right)^{3}} e^{-v_{1}^{2} / v_{0}^{2}} e^{-v_{2}^{2} / v_{0}^{2}} \sigma_{T}\left(\left|\vec{v}_{1}-\vec{v}_{2}\right|\right) \\
& =\int_{0}^{2 \sqrt{2} v_{0}} \frac{d^{3} v}{\left(2 \pi v_{0}^{2}\right)^{3 / 2}} e^{-v^{2} / 2 v_{0}^{2}} \sigma_{T}(v) .
\end{aligned}
$$

Note that although the escape velocity may be an underestimate here, increasing it by a factor of 10 changes $\left\langle\sigma_{T}\right\rangle$ only at the $1 \%$ level.

The thermally-averaged transfer cross section, then, depends on four parameters: $m_{X}, \Lambda, \alpha$, and $V_{\max }$. In Fig. 4, we plot the ratio $\left\langle\sigma_{T}\right\rangle / m_{X}$ in the $\left(m_{X}, \Lambda\right)$ plane for $\alpha=1$ and three representative characteristic velocities: $V_{\max }=40 \mathrm{~km} / \mathrm{s}$ for dwarfs, $V_{\max }=100 \mathrm{~km} / \mathrm{s}$ for LSBs, and $V_{\max }=1000 \mathrm{~km} / \mathrm{s}$ for clusters. For masses $m_{X} \sim 1 \mathrm{TeV}$ and $\Lambda \sim 10 \mathrm{MeV}$, we achieve transfer cross sections around the targeted range between $0.1 \mathrm{~cm}^{2} / \mathrm{g}$ and $1.0 \mathrm{~cm}^{2} / \mathrm{g}$ for all three systems under consideration. The transfer cross section decreases as a function of $v$ in the classical regime; 


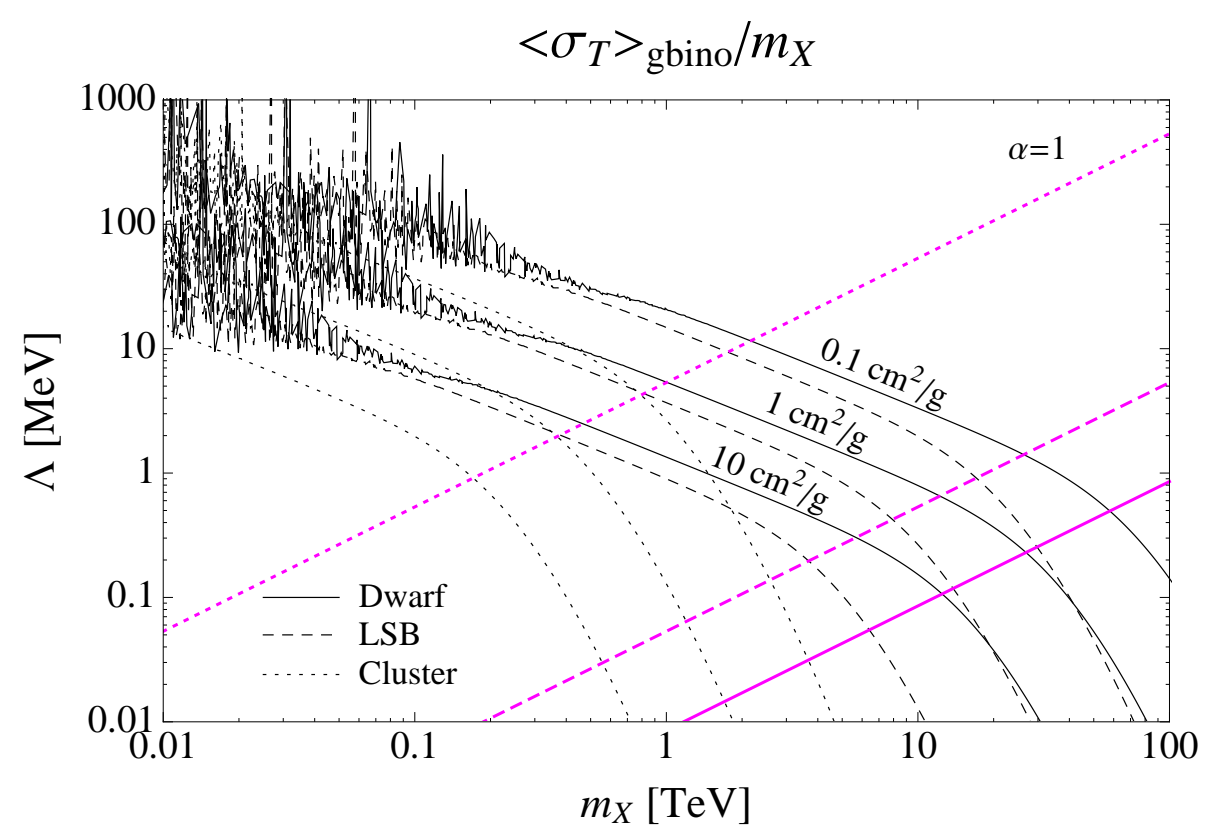

FIG. 4: The ratio of the thermally-averaged transfer cross section to dark matter mass $\left\langle\sigma_{T}\right\rangle / m_{X}$ in the $\left(m_{X}, \Lambda\right)$ plane for $\alpha=1$ and three different astrophysical systems: dwarf galaxies $\left(V_{\max }=40 \mathrm{~km} / \mathrm{s}\right.$, solid $)$, $\operatorname{LSBs}\left(V_{\max }=100 \mathrm{~km} / \mathrm{s}\right.$, dashed $)$, and clusters $\left(V_{\max }=1000 \mathrm{~km} / \mathrm{s}\right.$, dotted $)$. For each system, three values of the cross section are shown: $0.1 \mathrm{~cm}^{2} / \mathrm{g}$ (top), $1 \mathrm{~cm}^{2} / \mathrm{g}$ (middle), and $10 \mathrm{~cm}^{2} / \mathrm{g}$ (bottom). The region above the straight magenta lines show where inelastic processes may modify the picture based on elastic scattering for each type of system.

thus, systems with larger characteristic velocities have smaller cross sections, all else being equal. The LSB line at $0.1 \mathrm{~cm}^{2} / \mathrm{g}$, for instance, lies below that for dwarfs, because a larger interaction range (smaller $\Lambda$ ) is needed to counter its larger velocity to give the same $\sigma_{T}$ as the dwarfs. Toward the lower values of $m_{X}$, the scattering exhibits resonant behavior due to the formation of quasi-bound states [82], analogous to Sommerfeld enhancements in annihilations.

The region below the straight magenta lines in Fig. 4 is where the dark matter typically has $(1 / 2) m_{X} v^{2}>\Lambda$, and modifications from inelastic scattering processes can be important. We urge the reader to keep in mind that while in this region the classical elastic scattering cross section (for our assumed Yukawa potential) falls below about $3 \pi / \Lambda^{2}$, we expect other energy-exchange mechanisms to become important in dark matter halos. Note that for clusters $\left(v \sim 3 \times 10^{-3}\right)$, this is a substantial region of the interesting parameter space: $\left(m_{X} / \mathrm{TeV}\right) \gtrsim(\Lambda / 10 \mathrm{MeV})$. This suggests that the elastic glueballino scattering curves plotted for clusters in Fig. 4 and other figures are far from the whole story. We expect new astrophysical phenomenology, especially in clusters of galaxies, and this deserves separate consideration.

\section{GLUEBALLINO RELIC DENSITY}

One goal of supersymmetrizing the pure gauge hidden sectors considered in Sec. III is to revive the possibility of dark matter with naturally the right relic density, as in the case of WIMPs, but now for self-interacting dark matter. In this section, we first review the machinery required to calculate a glueballino relic density from the freezeout of thermal relic gluinos. We then discuss the possibility of realizing the correct thermal relic density through the WIMPless miracle in AMSB models [12]. 


\section{A. Gluino Freezeout}

In a supersymmetric pure gauge hidden sector, the gluinos are initially in equilibrium with a thermal bath of gluons at hidden sector temperature $T^{h}$. As the Universe cools below the gluino mass $m_{X}$, however, the gluinos freeze out. The gluino is the lightest supersymmetric particle in the hidden sector, and we will assume it is stable. In the absence of couplings to the visible sector, stability is guaranteed by Lorentz symmetry, as the gluino is the only fermion in the hidden sector.

The gluino relic density is determined by the usual thermal freezeout analysis, but with the slight extra complication that it occurs in a hidden sector with a temperature that may differ from the visible sector. For $S$-wave annihilation, the relic density of a thermal relic in a hidden sector is [11]

$$
\Omega_{X} \approx \frac{s_{0}}{\rho_{c 0}} \frac{3.79 x_{f}}{\left(g_{* S} / \sqrt{g_{*}^{\text {tot }}}\right) M_{\mathrm{Pl}}\langle\sigma v\rangle},
$$

where $s_{0}$ is the entropy of the visible sector today, $x_{f} \equiv m_{X} / T_{f}, \rho_{c 0}$ is the critical density today, and $g_{*}^{\text {tot }}=g_{*}+\xi_{f}^{4} g_{*}^{h}$ at freezeout.

We now discuss the various quantities entering Eq. (10). For the annihilation process $\tilde{g} \tilde{g} \rightarrow g g$, we use an $S$-wave cross section

$$
\langle\sigma v\rangle=k_{N} \frac{\pi \alpha_{X}^{2}}{m_{X}^{2}}
$$

where $\alpha_{X}=g_{h}^{2}\left(m_{X}\right) / 4 \pi$ is the fine-structure constant with a corresponding hidden-gauge coupling $g_{h}$ evaluated at the scale $m_{X}$, and $k_{N}$ is an $\mathcal{O}(1) N$-dependent coefficient, which we simply set to 1. Additionally, we set $x_{f}=25 \xi_{f}$, which is a good approximation for a large set of parameters [11]. Given this, $\Omega_{X}$ scales approximately linearly with $\xi_{f}$. The latest Planck results give a value of $\Omega_{\mathrm{DM}} h^{2}=0.1196 \pm 0.0031$ from a six-parameter fit to the $\Lambda \mathrm{CDM}$ model [24].

To determine the number of relativistic degrees of freedom in the visible and hidden sectors, note that, although the hidden and visible sectors need to interact gravitationally, they do not necessarily have to communicate otherwise, even at high energies. Thus, the sectors' temperatures are generically unrelated, and the ratio $\xi=T^{h} / T$ parameterizes this difference. The comoving entropies in the visible and hidden sector are independently conserved, and the values of $\xi$ at different times $t_{i}$ and $t_{f}$ are related by

$$
\frac{g_{* S}^{h}\left(t_{i}\right)}{g_{* S}\left(t_{i}\right)} \xi_{i}^{3}=\frac{g_{* S}^{h}\left(t_{f}\right)}{g_{* S}\left(t_{f}\right)} \xi_{f}^{3} .
$$

The effective number of relativistic degrees of freedom associated with the entropy (energy) density in the visible and hidden sectors are $g_{* S}$ and $g_{* S}^{h}\left(g_{*}\right.$ and $\left.g_{*}^{h}\right)$. As we will see, for most of the parameter space of interest, the gluinos freeze out at visible-sector temperatures at or above $T_{\mathrm{SM}} \approx$ $300 \mathrm{GeV}$, so that all SM particles are relativistic and $g_{* S}=g_{*}=106.75$. Although there may be minimal supersymmetric standard model (MSSM) superpartners with low enough masses to contribute to $g_{*}$ at freezeout, we assume the contribution is negligible, with most of the visible supersymmetric-partner spectrum being above $m_{X}$. For the gluons and gluinos,

$$
g_{*}^{h}=g_{* S}^{h}= \begin{cases}4\left(N^{2}-1\right) & T^{h} \gtrsim m_{X} \\ 2\left(N^{2}-1\right) & m_{X} \gtrsim T^{h}>\Lambda .\end{cases}
$$




\section{B. The WIMPless Miracle and AMSB}

As noted above, the gluino thermal relic density has the parametric dependence

$$
\Omega_{X} \sim \frac{1}{\langle\sigma v\rangle} \sim \frac{m_{X}^{2}}{\alpha_{X}^{2}} .
$$

For weak-scale masses and weak interaction coupling strengths, $\Omega_{X}$ is of the desired size; this is the essence of the WIMP miracle. For the hidden sector, we have great freedom in choosing the parameters $m_{X}$ and $\alpha_{X}$, and may simply choose them to yield the correct relic density. However, it is preferable if the correct mass-to-coupling ratio is set in a non-contrived way. This is a property of models that realize the WIMPless miracle $[10,11]$, where the dark matter's mass and coupling are not fixed individually, but the ratio $m_{X}^{2} / \alpha_{X}^{2}$ is fixed to the desired value by the model framework.

Supersymmetric models with AMSB $[8,9]$ provide a particularly nice realization of the WIMPless miracle [12-14]. In AMSB, the MSSM is sequestered from the supersymmetry-breaking sector, so the gaugino masses in the visible sector do not receive any tree-level contributions and are instead generated by the Weyl anomaly, leading to

$$
m_{v} \sim \frac{\alpha_{v}}{4 \pi} m_{3 / 2}
$$

where $m_{3 / 2}$ is the gravitino mass, $\alpha_{v}$ is a SM fine-structure constant, and $m_{v}$ is of the order of the weak scale, if these models are to address the gauge hierarchy problem. In any additional sequestered hidden sector of the theory, the hidden sector superpartner masses will be given by a similar relation,

$$
m_{X} \sim \frac{\alpha_{X}}{4 \pi} m_{3 / 2}
$$

where $\alpha_{X}$ is the hidden sector's fine-structure constant. Since there is only one gravitino mass, $m_{X} / \alpha_{X} \sim m_{v} / \alpha_{v}$, and any hidden sector thermal relic in AMSB can be expected to have the desired relic density, even if $m_{X}$ and $\alpha_{X}$ differ, perhaps greatly, from the SM values.

The visible sector of AMSB models contains a stable thermal relic, the lightest neutralino. However, the standard AMSB relations imply that this is the Wino. Winos annihilate very efficiently, and must have masses around $2.7-3.0 \mathrm{TeV}$ to be all of dark matter [88, 89]. The thermal relic density scales as $\sim m_{\tilde{W}}^{-2}$, and so for lighter and more natural values closer to the LEP2 experimental limit $m_{\tilde{W}} \gtrsim 100 \mathrm{GeV}$ [90, 91], the Wino's thermal relic density is completely negligible. We will therefore neglect it below, and take this as additional motivation to develop AMSB models with viable hidden sector dark matter candidates.

The particle spectrum in AMSB models is completely specified by quantum numbers, dimensionless couplings, and the gravitino mass. In the visible sector, the Wino mass limit $m_{\tilde{W}} \gtrsim 100 \mathrm{GeV}$ implies

$$
m_{3 / 2} \gtrsim 37 \mathrm{TeV}
$$

In the hidden sector, at scales above $m_{X}$, the one-loop $\beta$-function coefficient is $b_{H}=-3 N$, and the theory is asymptotically free. The gluino mass is

$$
m_{X}=-b_{H} \frac{\alpha_{X}}{4 \pi} m_{3 / 2}=3 N \frac{\alpha_{X}}{4 \pi} m_{3 / 2}
$$

Below $m_{X}$, we have a non-supersymmetric $\mathrm{SU}(N)$ gauge theory with a $\beta$-function coefficient $b_{L}=$ $-(11 / 3) N$. The theory is expected to confine at the scale

$$
\Lambda \sim m_{X} \exp \left(\frac{-6 \pi}{11 N \alpha_{X}}\right)=m_{X} \exp \left(\frac{-9 m_{3 / 2}}{22 m_{X}}\right) .
$$


With this relationship, the relic density in Eq. (10) becomes

$$
\Omega_{X} \approx \frac{s_{0}}{\rho_{c 0}} \frac{\left[g_{*}+2\left(N^{2}-1\right) \xi_{f}^{4}\right]^{1 / 2}}{g_{* S}} \frac{3.79 \cdot 25 \xi_{f}}{M_{\mathrm{Pl}}} \frac{9 N^{2}}{16 \pi^{3}} m_{3 / 2}^{2} .
$$

\section{GLUEBALLINO/GLUEBALL DARK MATTER WITHOUT CONNECTORS}

Given the results above, we can now present simple AMSB models of self-interacting dark matter. We begin by considering the simple case without connector fields, in which the visible and hidden sectors are decoupled. The visible sector is the MSSM; the tachyonic slepton problem is assumed to be solved in a way that does not impact the masses of the MSSM gauginos, and the Wino is assumed to be the visible LSP, with negligible thermal relic density. The hidden sector is a pure $\mathrm{SU}(N)$ gauge theory, consisting of gluinos and gluons, which confine to form glueballino and glueball dark matter.

There are only four independent parameters in the theory, which may be taken to be

$$
m_{X}, \Lambda, N, \xi_{f}
$$

These determine $\alpha_{X}$ and $m_{3 / 2}$ through Eq. (19). In contrast to the model-independent discussion of Sec. IV, in AMSB models, renormalization group equations relate the high-scale parameters $m_{X}$ and $\alpha_{X}$ to the low-scale parameter $\Lambda$. In terms of these parameters, the glueball self-interaction cross section and relic density are determined as described in Secs. III A and III B, and the glueballino self-interaction cross section and relic density are determined as described in Secs. IV and V.

We first present results for models with mostly-glueballino dark matter in Fig. 5. We scan over the $\left(m_{X}, \Lambda\right)$ plane. At every point in this plane, we require that glueballinos make up $90 \%$ (top panel) or $99.99 \%$ (bottom panel) of the dark matter, and glueballs make up the remaining $10 \%$ or $0.01 \%$. The constraints on $\Omega_{\text {gbino }}$ and $\Omega_{\mathrm{gb}}$ determine $N$ and $\xi_{f}$; contours of constant $N$ and $\xi_{f}$ are shown. The lower bound of Eq. (17) excludes parameter space with low $m_{X}$. In the remaining parameter space, $m_{X} / \Lambda \gtrsim 10^{3}$, more than sufficient to ensure $T_{f}^{h}>\Lambda$, so gluino freezeout occurs in the weakly-interacting theory, and the thermal freezeout calculation is valid.

These relic density results for a particular glueballino density may be understood as follows. On a given curve of constant $N$, larger dark matter masses imply larger thermal relic densities and so require smaller values of $\xi_{f}$ to keep $\Omega_{\text {gbino }}$ fixed. Once $\xi_{f}$ decreases, a larger $\Lambda$ is required to keep $\Omega_{\mathrm{gb}}$ constant. Note also that for $\Lambda \sim \mathrm{MeV}$ and $\xi_{f} \sim 1$, glueballs overclose the Universe. To avoid this, $\xi_{f}$ must be lowered, and to have mostly-glueballino dark matter, $m_{X}$ must be a bit larger than the weak scale. In short, the presence of glueballs forces the model away from the $a$ priori most natural parameter space with low $m_{X}$ and $\xi_{f} \sim 1$. In the context of AMSB, however, it is rather natural to assume that the hidden and visible sectors are separated at high scales and

$\xi_{f} \ll 1$. Given this, the WIMPless miracle is nicely realized in regions of parameter space with $\xi_{f} \sim 0.01$ and $N \sim \mathcal{O}(1)$ for $\Omega_{\text {gbino }}=0.9 \Omega_{\mathrm{DM}}$.

There are also differences between the $90 \%$ and $99.99 \%$ glueballino cases. The curves of constant $N$ and constant $\xi_{f}$ shift as the relative amounts of glueball and glueballino dark matter change. By focusing on a particular point in the $\left(m_{X}, \Lambda\right)$ plane and comparing Eq. (4) and Eq. (20), we find

$$
\xi_{f} \sim \frac{\Omega_{\mathrm{gb}}^{1 / 2}}{\Omega_{\mathrm{gbino}}^{1 / 2}} \quad \text { and } \quad N \sim \frac{\Omega_{\mathrm{gbino}}^{3 / 4}}{\Omega_{\mathrm{gb}}^{1 / 4}}
$$



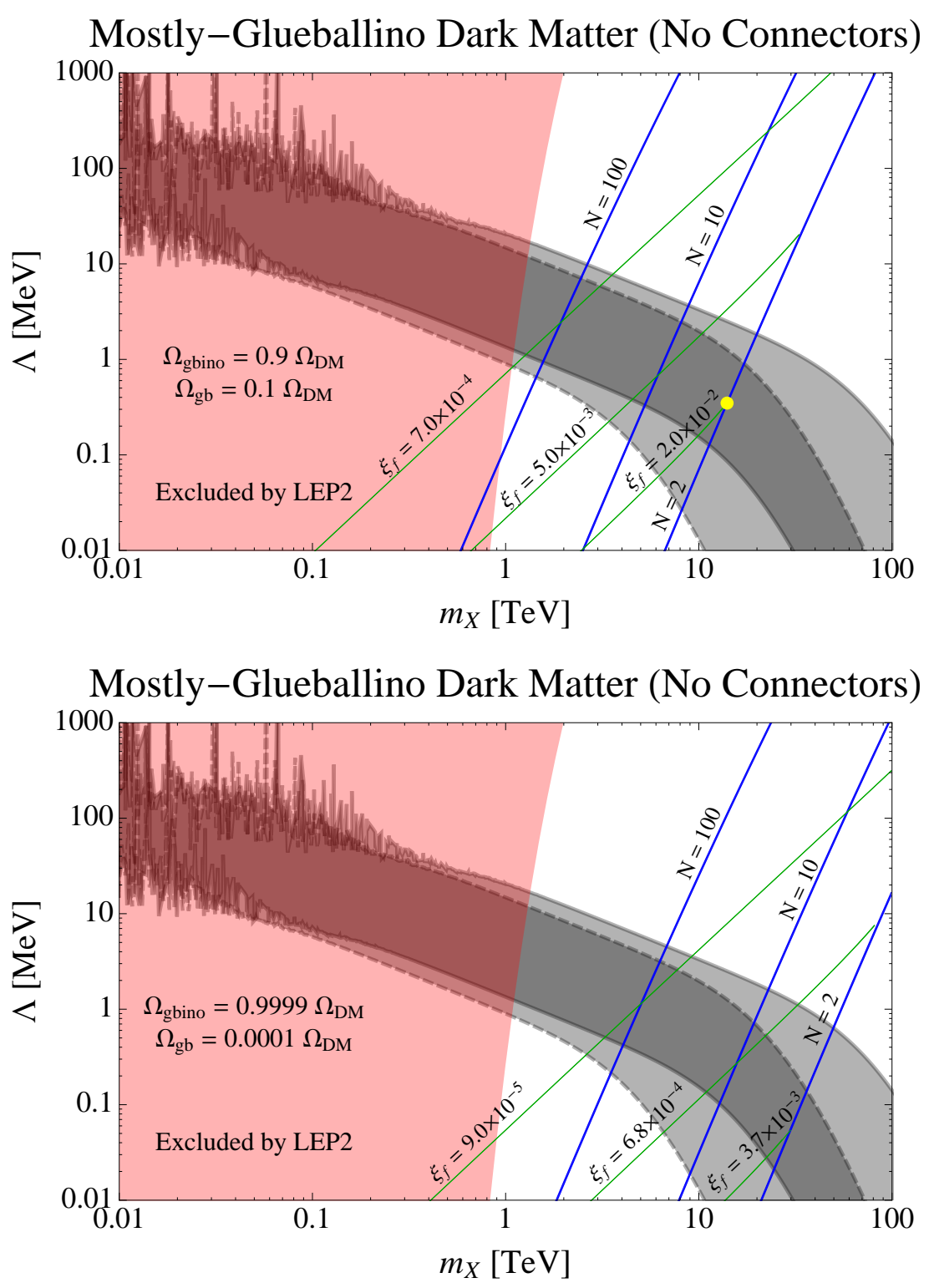

FIG. 5: Mostly-glueballino dark matter in AMSB models with pure SU(N) hidden sectors without connectors. Glueballinos make up $90 \%$ (top) or $99.99 \%$ (bottom) of the dark matter, and glueballs make up the remaining portion. For a point in the $\left(m_{X}, \Lambda\right)$ plane, these constraints on the relic densities determine $N$ and $\xi_{f}$; contours of constant $N$ and $\xi_{f}$ are shown. The gray, shaded bands are from Fig. 4 and give the regions where the glueballino self-interaction cross section is in the preferred range. The red, shaded region is excluded by null searches for visible-sector Winos at LEP2. The yellow dot in the left panel defines a representative model with $m_{X} \simeq 14 \mathrm{TeV}, \Lambda \simeq 0.35 \mathrm{MeV}, N=2$, and $\xi_{f} \simeq 0.02$.

for $N^{2} \gg 1$. When the glueball density is reduced by 3 orders of magnitude, we expect $N$ to increase by a factor of $10^{3 / 4} \sim 6$ and $\xi_{f}$ to decrease by a factor of $10^{3 / 2} \sim 30$; this can be seen in Fig. 5.

Of course, the goal is not simply to obtain a multi-component model of dark matter with the correct relic densities, but to obtain self-interacting dark matter. The regions with the preferred self-interaction cross sections are also shown in Fig. 5. For values of $m_{X} \sim 10 \mathrm{TeV}, \Lambda \sim 1 \mathrm{MeV}$, $2 \leq N \lesssim 10$ and $10^{-3} \lesssim \xi_{f} \lesssim 10^{-2}$, we find models that satisfy the relic density constraints and also satisfy the scattering constraints for dwarfs and LSBs. Viable models also exist for lower $m_{X}$ down 
to the LEP2 limit for larger $N$ and lower $\xi_{f}$. A representative model is one with $m_{X} \simeq 14 \mathrm{TeV}$, $\Lambda \simeq 0.35 \mathrm{MeV}, N=2$ and $\xi_{f} \simeq 0.02$; this is shown as a yellow dot in Fig. 5. For these parameters, Fig. 1 shows how the dark matter coupling behaves from the scale $m_{X}$ down to confinement.

Measurements of nuclei abundances and of the CMB place restrictions on the number of light degrees of freedom $N_{\text {eff }}$ around the time of BBN that contribute to the expansion of the Universe. Results from Planck give $N_{\text {eff }}=3.30 \pm 0.27$ [24]. An interesting question, then, is whether these models also imply non-standard values of $N_{\text {eff }}$. Once the hidden-sector temperature drops below the confinement scale, glueballinos and glueballs form. This occurs when the visible sector's temperature is

$$
T_{\Lambda}=\frac{T_{\Lambda}^{h}}{\xi_{\Lambda}} \sim \frac{\Lambda}{\xi_{\Lambda}}=\frac{\Lambda}{\xi_{f}}\left(\frac{g_{* S}\left(t_{f}\right)}{g_{* S}\left(t_{\Lambda}\right)}\right)^{1 / 3}
$$

using Eq. (12) with $g_{* S}^{h}\left(t_{\Lambda}\right)=g_{* S}^{h}\left(t_{f}\right)$. For the representative example parameters given above, the confinement scale is $T_{\Lambda} \sim 90 \mathrm{MeV}$; confinement occurs well before BBN and structure formation, as expected. There is therefore no relativistic, massless species to act as the hidden sector bath during $\mathrm{BBN}$. At the time of $\mathrm{BBN}$, the hidden-sector temperature is not well defined, and its contribution to $N_{\text {eff }}$ is essentially zero.

We next consider the case of mostly-glueball dark matter. To be concrete, we present the case of $\Omega_{\mathrm{gb}}=0.9 \Omega_{\mathrm{DM}}$ and $\Omega_{\mathrm{gbino}}=0.1 \Omega_{\mathrm{DM}}$ in Fig. 6 . Once again, we show contours of constant $N$ and $\xi_{f}$, but now we include the glueball scattering constraints from Fig. 3, since glueballs are the dominant component of dark matter. The values of $m_{X}$ that satisfy relic and scattering constraints for a given $N$ are fairly similar to those in the case of mostly-glueballino dark matter; however, the corresponding values of $\Lambda$ are a few orders of magnitude larger than the mostly-glueballino case.

In Figs. 5 and 6 , the fraction of glueballino to glueball dark matter is fixed. Of course, different values are possible. In Fig. 7, we fix $N=2$ and vary $m_{X}$ and $\Lambda ; \xi_{f}$ is set by the requirement that $\Omega_{\mathrm{gbino}}+\Omega_{\mathrm{gb}}=\Omega_{\mathrm{DM}}$. The results are presented in the $\left(\left\langle\sigma_{T}\right\rangle_{\text {gbino }} / m_{X},\left\langle\sigma_{T}\right\rangle_{\mathrm{gb}} / \Lambda\right)$ plane, where $V_{\max }=40 \mathrm{~km} / \mathrm{s}$, and contours of constant $\Omega_{\mathrm{gbino}} / \Omega_{\mathrm{gb}}$ are shown. Regions excluded by LEP2 and by cluster bounds are shaded.

Figure 7 shows that the fraction of dark matter that is glueballinos may take almost any value in the parameter space. Of course, regions of parameter space that are overwhelmingly glueballino-dominated and have too-large glueballino self-interactions are excluded, as are regions that are overwhelmingly glueball-dominated with too-large glueball self-interactions. The parts of parameter space that are certainly excluded by these considerations are indicated, but the position of this boundary is somewhat uncertain and requires detailed $N$-body simulations (modeling both components of dark matter) to determine. The cluster constraints $[2,65]$ are relevant here because glueballs have a velocity-independent scattering cross section and these constraints dictate that glueballs must be the subdominant component of dark matter in all of the parameter space shown in Fig. 7.

Especially interesting, however, are the regions of parameter space with a subdominant component of dark matter that self-interacts very strongly. For example, the dark matter may be $99 \%$ glueballinos and $1 \%$ glueballs, but the glueballs may have $\left\langle\sigma_{T}\right\rangle_{\mathrm{gb}} / \Lambda \sim 10^{5}-10^{11} \mathrm{~cm}^{2} / \mathrm{g}$. Such possibilities are not ruled out by the constraints discussed so far but may have very interesting astrophysical implications.

It has been pointed out that, at early times before the halo has had time to form a core through self-interactions, seed black holes can grow by accreting self-interacting dark matter [92]. In the mixed self-interacting dark matter scenario where one of the components has $\left\langle\sigma_{T}\right\rangle / \mathrm{m} \gg 1 \mathrm{~cm}^{2} / \mathrm{g}$, this accretion can be highly enhanced. The possibility that supermassive black hole growth is seeded by the self-interactions of either the glueballs or glueballinos is an exciting prospect. There 

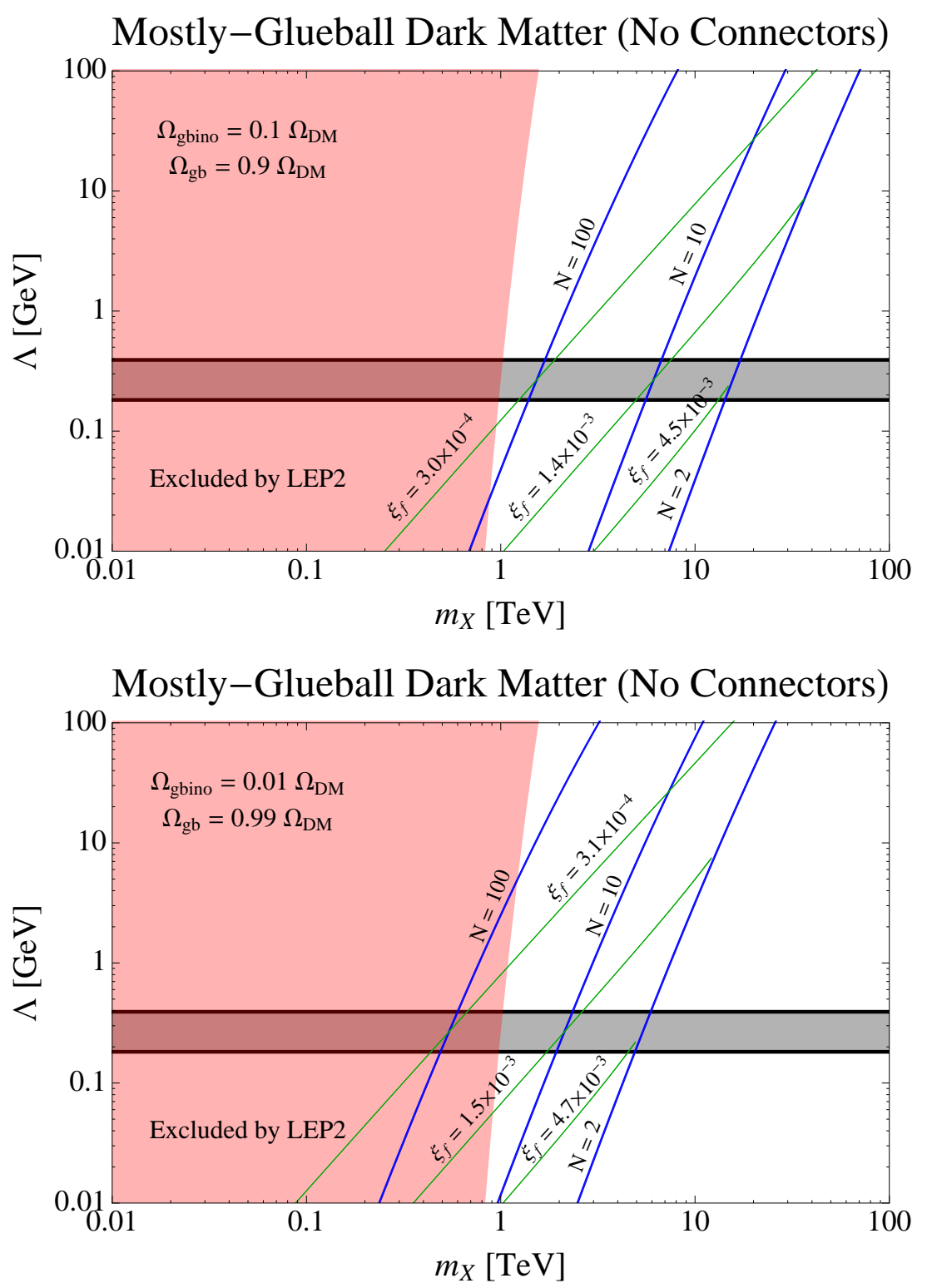

FIG. 6: Mostly-glueball dark matter in AMSB models with pure SU(N) hidden sectors and no connectors. Glueballs make up $90 \%$ (top) or $99 \%$ (bottom) of the dark matter, and glueballinos make up the remaining portion. For a point in the $\left(m_{X}, \Lambda\right)$ plane, these constraints on the relic densities determine $N$ and $\xi_{f}$; contours of constant $N$ and $\xi_{f}$ are shown. The gray, shaded band is from Fig. 3 and gives the region where the glueball self-interaction cross section is in the preferred range. The red, shaded region is excluded by null searches for visible-sector Winos at LEP2.

is not yet a clear picture of how $10^{9} \mathrm{M}_{\odot}$ quasars are assembled already by $z \gtrsim 6$ within the standard $\Lambda \mathrm{CDM}$ cosmology. Models starting with the expected $100 \mathrm{M}_{\odot}$ seeds require special assumptions about the mass accretion histories of these quasars [93], which become more strained as higher redshift quasars are found [94]. Self-interactions within the dark matter sector may have a big role to play in this story, as they generically enhance the early black hole accretion rate.

There is a tight correlation between the mass of supermassive black holes in the centers of galaxies and the velocity dispersion or luminosity of the bulge [96]. By requiring that the predicted masses of supermassive black holes are not overly large, it should also be possible to constrain the ratio $\Omega_{\mathrm{gb}} / \Omega_{\text {gbino }}$ in mixed self-interacting dark matter models where $\left\langle\sigma_{T}\right\rangle_{\mathrm{gb}} / \Lambda$ is large. To 


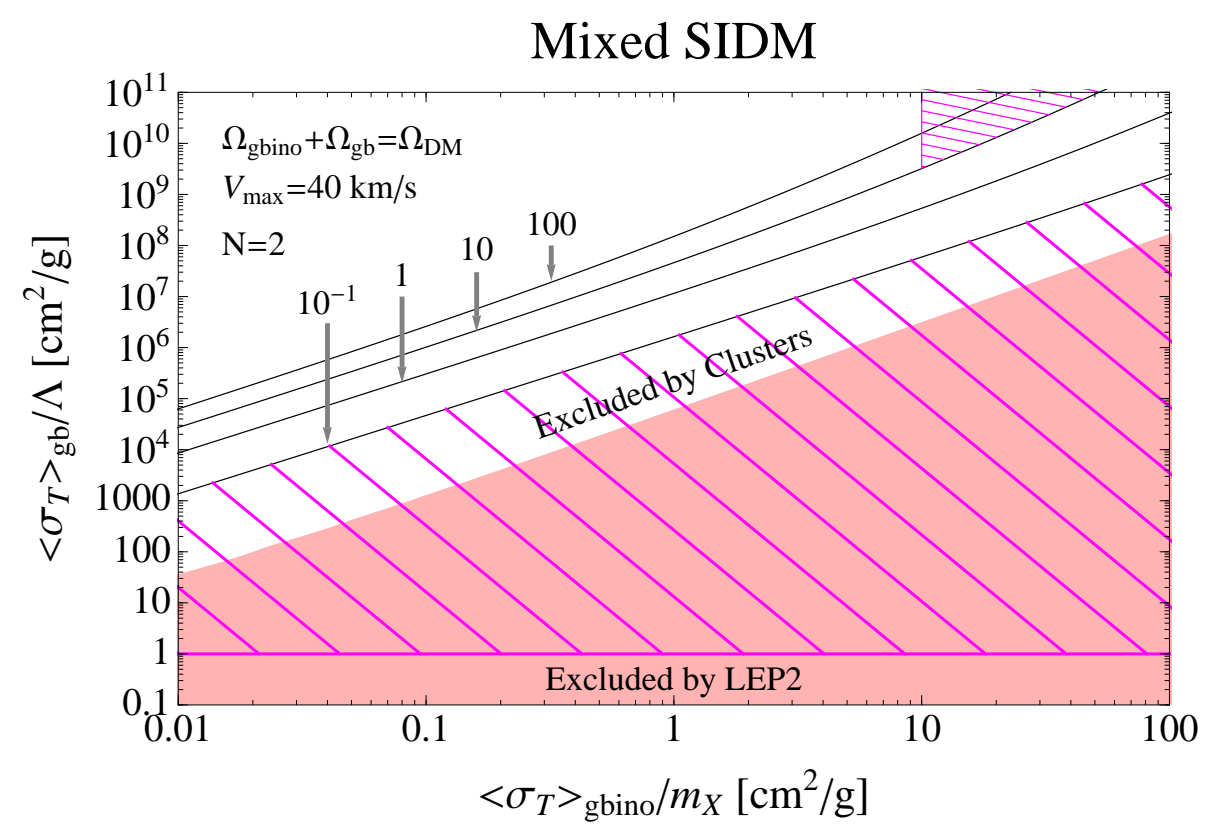

FIG. 7: Mixed dark matter without connectors to the Standard Model. We show curves of constant $\Omega_{\mathrm{gbino}} / \Omega_{\mathrm{gb}}$ in the $\left(\left\langle\sigma_{T}\right\rangle_{\text {gbino }} / m_{X},\left\langle\sigma_{T}\right\rangle_{\mathrm{gb}} / \Lambda\right)$ plane, for fixed $N=2$ and considering dwarf systems with $V_{\max }=40 \mathrm{~km} / \mathrm{s}$. The black curves have $\Omega_{\mathrm{gbino}} / \Omega_{\mathrm{gb}}=0.1,1,10,100$, as indicated. The bound from LEP2 is shown in the red, shaded region. A stronger bound from clusters is shown in the lower magenta, hatched region; since the glueball scattering cross section is the same on all scales, its value is limited for the dwarf systems to avoid violating bounds from cluster scales. We caution the reader that the bound may be stronger and it is certainly not as sharp as indicated by the hatched region. The magenta hatched wedge near the upper right-hand portion of the graph represents an upper limit of $10 \mathrm{~cm}^{2} / \mathrm{g}$ for the case of mostly-glueballino dark matter, which will have important implications for cores in dwarfs galaxies and may be excluded by a comparison to the observed core sizes and densities (e.g., Ref. [1, 95]).

correctly implement this constraint, many new features of our simple model and their astrophysical consequences will have to be worked out. We highlight a few of these below.

The details of capture of glueballs by a seed black hole will differ significantly from the treatment in Ref. [92]. The black hole capture depends sensitively on the density profile of glueballs, and this is tightly correlated with the potential well of the galaxy, which is dominated by glueballinos. In particular, although an isolated strongly self-interacting dark matter halo will undergo core collapse, this is not true when the strongly self-interacting component (glueballs) is a small fraction of the dark matter.

A complicating factor is that the glueballs and glueballinos will scatter off of each other. Each collision will change the velocity of glueballs by $\mathcal{O}(1)$, but the velocity of glueballinos will only change by $\Lambda / m_{X} \ll 1$. The glueballino-glueball scattering cross section should be of the order the geometric cross section $\left(\sim 1 / \Lambda^{2}\right)$, and thus, this could be an important effect if the number density of glueballinos is much larger than that of glueballs (either because of a small $\Omega_{\mathrm{gb}} / \Omega_{\mathrm{gbino}}$ or as glueballs are depleted due to accretion by the black hole). Conversely, this scattering could also have an impact on the glueballino density profile if the number density of glueballs is large enough to overcome the small momentum transfer.

Another important effect, relevant for halo properties as well as black hole growth, is cooling. We have focused on elastic collisions in this paper, but as mentioned previously there are also inelastic processes leading to cooling through the emission of glueballs. Cooling will funnel more glueballs into the inner regions (modulo angular momentum constraints) and increase the black 
hole accretion rate. Note that unlike the baryons, competing effects from star formation and subsequent heating by UV photons are not relevant for glueballs.

As an extreme example, one could assume that all of the glueballs are bound up in the central supermassive black hole. In this case, we can use measured ratios of the black hole masses to halo

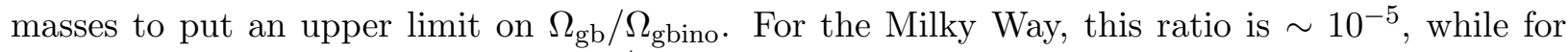
Andromeda the ratio is more like $10^{-4}$. (It should be kept in mind that the black hole will also accrete baryons and grow, so this is a lenient upper bound.) Rather than focus on the Local Group, one could look more generally at the black hole mass-virial mass relation for all galaxies, but as expected there is a lot of scatter in this relation [97].

To illustrate the effect of these constraints on the model parameter space, we have shown two possibilities in Fig. 5: one with $\Omega_{\mathrm{gb}} / \Omega_{\mathrm{gbino}}=0.1$ (which may not be viable given the arguments above) and a second with $\Omega_{\mathrm{gb}} / \Omega_{\mathrm{gbino}}=10^{-4}$. There is no impediment in making this ratio even smaller, although there is no natural reason to do so. In addition, as $\Omega_{\mathrm{gb}} / \Omega_{\mathrm{gbino}}$ is reduced, the regions with small $N$ move into the regime where inelastic process will be important for all relevant velocities (dwarfs to clusters).

\section{GLUEBALLINO/GLUEBALL DARK MATTER WITH CONNECTORS}

Although a pure $\mathrm{SU}(N)$ hidden sector with no connectors can accommodate both early Universe and structure formation constraints, it is interesting to consider the possibility of connector fields that allow communication between the hidden and visible sectors. Such scenarios may have, of course, a larger number of testable implications. In addition, as we will see, if the connectors mediate annihilation or decays to the visible sector, the viable parameter space may be significantly altered.

If the hidden and visible sectors communicate, we expect the temperatures of the two sectors to coincide nearly until kinetic decoupling at confinement. If glueballs are stable, they will generically overclose the Universe, and so there must be a mechanism to reduce the glueball density. Let us assume that this mechanism exists and reduces the glueball relic density to a negligible level. We can then immediately determine the consequences for the parameter space. For a given point in the $\left(m_{X}, \Lambda\right)$ plane with $\xi_{f}=1$, there are contours of fixed $N$ on which $\Omega_{\text {gbino }}=\Omega_{\mathrm{DM}}$. These are shown in Fig. 8, along with the self-interaction constraints. We see that the LEP2 bound excludes all but the $N \leq 4$ possibilities, but now, for low $N$, the allowed values of $m_{X}$ are much reduced and more natural relative to the case without connectors.

A straightforward way to eliminate glueballs is through decays, but other constraints render this scenario unacceptable. The glueballs have a mass around 1 to $10 \mathrm{MeV}$, so possible decay products will be photons, electrons, and neutrinos. Decays to photons will typically take too long and happen well after BBN. If too much energy and entropy is injected into the visible sector at $T \lesssim 1 \mathrm{MeV}$, then there is an unacceptably large contribution to $N_{\text {eff. }}$. Decays to electrons after $1 \mathrm{MeV}$ face a similar problem, and in addition, they can break up deuterium and ruin its BBN abundance (if the glueball is heavy enough). Decays to light neutrinos are problematic, because glueballs can be produced in supernovae, escape the neutrino sphere, and cool the supernovae too efficiently. If we attempt to adjust parameters to get around the difficulties with either electrons or neutrinos, then we encounter problems with $e^{+} e^{-}$collider constraints. We are led to consider alternative processes to eliminate the glueball density.

Since decays after confinement are highly constrained, we investigate reducing the glueballino density by depleting the gluon density before confinement. The gluons may annihilate to SM particles via loop diagrams, but the reverse process needs to be suppressed. Let us introduce a right-handed neutrino $\nu_{R}$. The $\nu_{R}$ is a SM gauge singlet with a mass $m_{R} \sim \mathrm{GeV}$ and it could 


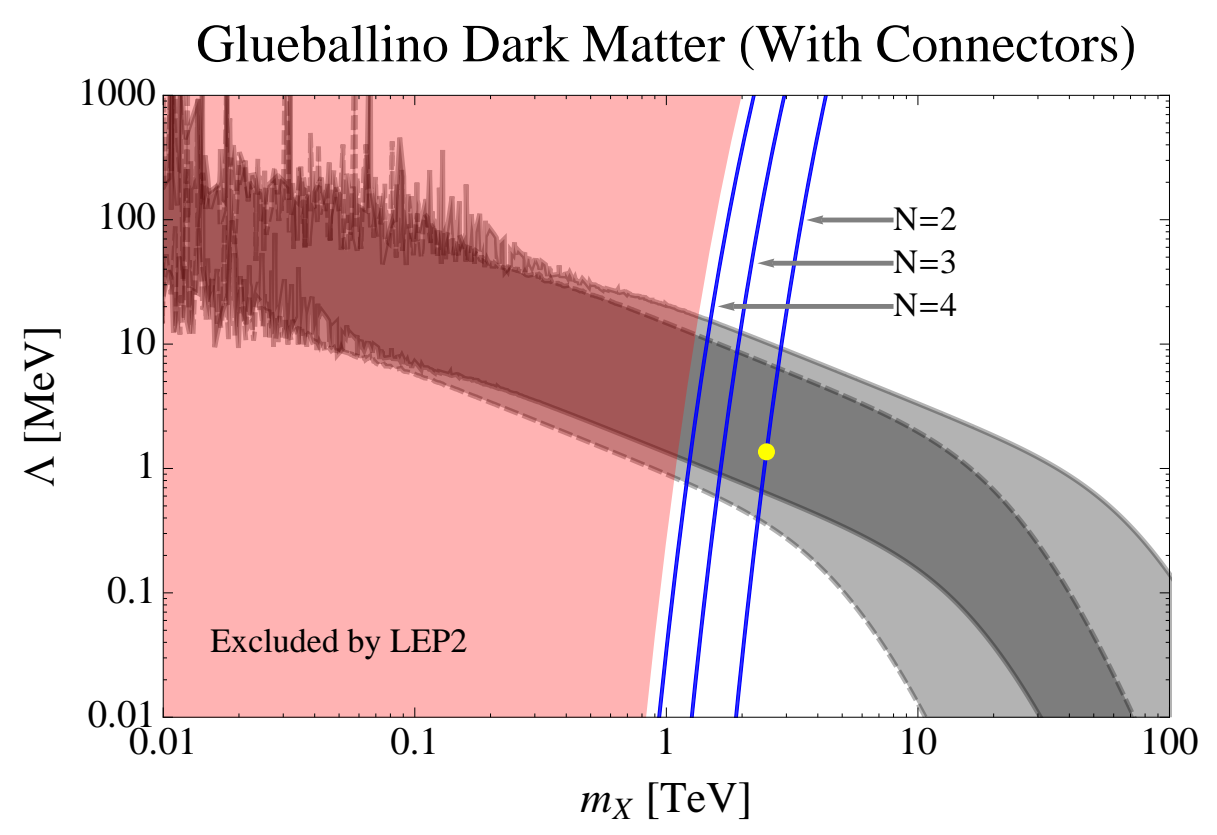

FIG. 8: Glueballino dark matter in AMSB models with pure $S U(N)$ hidden sectors and connectors to the $S M$. Glueballinos are assumed to make up all of the dark matter. The relic density constraints are given in the $\left(m_{X}, \Lambda\right)$ plane with $\xi_{f}=1$; contours of constant $N$ are shown. The gray, shaded bands are from Fig. 4 and give the regions where the glueballino self-interaction cross sections are in the preferred range. The red, shaded region is excluded by null searches for visible-sector Winos at LEP2. The yellow dot defines a representative model with $m_{X} \simeq 2.5 \mathrm{TeV}, \Lambda \simeq 1.4 \mathrm{MeV}$, and $N=2$.

be one of the sterile states in a see-saw mechanism to produce neutrino masses. Our goal is for the gluons to annihilate into right-handed neutrinos, which then decay quickly into SM particles before they can annihilate back into gluons.

To implement this scenario, we postulate that there is a connector field $C$ with mass $m_{C}$ that allows communication between the hidden and visible sector. The connector has a Yukawa interaction $\lambda_{R} C \bar{\nu}_{R} \nu_{R}$ in the visible sector and a gauge interaction with the gluons with strength $g_{h}$ in the hidden sector. Integrating out the connector produces the effective interaction

$$
\mathcal{L} \sim \frac{1}{16 \pi^{2}} \frac{\lambda_{R}^{2} g_{h}^{2}}{m_{C}^{3}} G_{\mu \nu}^{h} G^{h \mu \nu} \bar{\nu}_{R} \nu_{R}
$$

This interaction leads to an annihilation cross section,

$$
\langle\sigma v\rangle_{g g \rightarrow \bar{\nu}_{R} \nu_{R}} \sim \frac{\lambda_{R}^{4} g_{h}^{4}}{8 \pi\left(16 \pi^{2}\right)} \frac{T^{4}}{m_{C}^{6}} \equiv \sigma_{0} z^{-4},
$$

where $z=m_{R} / T$. Note that the annihilation of gluons into right-handed neutrinos is subdominant to the annihilation rate of gluons into gluinos and can be ignored in the gluino freezeout calculations. The right-handed neutrino decays with a rate

$$
\Gamma_{R} \sim \frac{g_{\nu}^{2}}{4 \pi} \frac{m_{R}^{2}}{T} \equiv \Gamma_{0} z
$$

into SM particles at tree level with a coupling strength $g_{\nu}$. As long as the neutrino decay rate is much faster than the gluon annihilation into neutrinos (and both are faster than the Hubble expansion), the gluons cannot maintain their equilibrium density, and their energy is transferred 
to SM particles. The depletion terminates no later than $\sim m_{R}$, when any surviving right-handed neutrinos freeze out, and the gluon density decreases subsequently only due to Hubble expansion.

To give a concrete example, consider the following parameters: $N=2, m_{X}=2.5 \mathrm{TeV}, \Lambda \simeq$ $1.4 \mathrm{MeV}, m_{C}=0.5 \mathrm{TeV}, m_{R}=1 \mathrm{GeV}, g_{h}=1.1, \lambda_{R}=1.6$, and $g_{\nu}=0.1$. The output glueball relic density is $\sim 5 \%$ of the total dark matter abundance. We find this result by numerically solving the coupled Boltzmann equations for the gluons and right-handed neutrinos:

$$
\begin{aligned}
Y_{g}^{\prime}(z) & =-z^{-6} \sigma_{0} \frac{s\left(m_{R}\right)}{H\left(m_{R}\right)}\left(Y_{g}^{2}-Y_{R}^{2}\right) \\
Y_{R}^{\prime}(z) & =-z^{-6} \sigma_{0} \frac{s\left(m_{R}\right)}{H\left(m_{R}\right)}\left(Y_{R}^{2}-Y_{g}^{2}\right)-z^{2} \frac{\Gamma_{0}}{H\left(m_{R}\right)} Y_{R},
\end{aligned}
$$

where $s\left(m_{R}\right)$ and $H\left(m_{R}\right)$ are the entropy and Hubble rate at $T=m_{R}$. The initial conditions $Y_{R}\left(z_{f}\right)$ and $Y_{g}\left(z_{f}\right)$ are given by Eq. (3) at dark matter freezeout, $z_{f}=25 m_{R} / m_{X}$. These differential equations tend to be fairly stiff, so in certain regions of parameter space, it is beneficial to decouple the equations. We may do so if the neutrino decay term dominates, allowing us to approximate $Y_{R}$ as exponentially decaying. Solving the decoupled differential equation yields results that are numerically similar (typically within 10\%) to solving the full set of coupled equations when the decay term dominates.

There are few constraints on this mechanism. Prior to confinement, a large amount of entropy is transferred from the gluons to light SM particles. Since the right-handed neutrinos are still relativistic, there is no entropy non-thermally deposited into the visible sector. All the righthanded neutrino decay products fall into equilibrium with the bath well before BBN. With a nonzero glueball density, a concern might be that the glueballs will be able to decay to SM particles via off-shell right-handed neutrinos and non-thermally deposit entropy into the visible sector. If the right-handed neutrino decays into a left-handed neutrino and the Higgs, then we expect the glueball decay rate into $\bar{\nu}_{L} \nu_{L} e^{+} e^{-} e^{+} e^{-}$to be

$$
\Gamma_{\mathrm{gb}} \sim y_{e}^{4} g_{\nu}^{4} \frac{\Lambda^{19}}{m_{C}^{6} m_{h}^{8} m_{R}^{4}},
$$

where $y_{e}$ is the electron Yukawa coupling and $m_{h}$ is the mass of the Higgs. This decay rate is slow enough that the glueballs are essentially stable and further will not contribute significantly to $N_{\text {eff }}$, since they are nonrelativistic below $1 \mathrm{MeV}$, and they will not have a large impact on the expansion rate of the Universe during BBN, given their small energy density. Our glueball depletion process is robust, and it is consistent with terrestrial and cosmic constraints [98].

\section{CONCLUSIONS}

We have explored the possibility that dark matter may be a composite particle, made up of bound states of a dark analogue of QCD in the hidden sector. Such constructions lead to rich and varied phenomena that are distinct from the WIMP scenario more typically considered. It also naturally leads to large self-interactions of the dark matter, which can explain several observational puzzles in the small scale structure of the Universe.

The simplest scenarios contain only dark gluons, which confine into glueballs with cosmologically interesting scattering cross sections for confinement scales around $100 \mathrm{MeV}$. Arranging the correct relic density requires one to disconnect the temperatures in the hidden and visible sectors such that their ratio at confinement is $\sim 10^{-3}$.

A richer theory arises when one considers supersymmetric versions, for which the dark gluino mass provides a separate mass scale and (in AMSB) can provide the correct relic density of glueballinos via the WIMPless miracle. The phenomenology depends crucially on how connected the 
hidden sector is to the visible matter. If there are no light connecting particles, one can dial the balance of dark matter from glueballs to glueballinos by adjusting the relative temperatures of the hidden and visible sectors. These mixed scenarios are strongly-interacting analogues of atomic dark matter [99-103] and inspire further simulation of the galactic dynamics in cases where there are two components of dark matter with naturally very different mass scales and different selfinteraction rates. Such simulations would be very helpful to better understand the observational limits on these theories. For clusters, another important issue is the fact that the dark matter may have enough energy to scatter inelastically, bringing the details of the dark composite sector to the forefront of the physics; further work is needed to better understand the implications. We have also pointed out that our models have rich implications for the early growth of supermassive black holes. The mechanism by which $\sim 10^{9} \mathrm{M}_{\odot}$ quasars are assembled as early as redshifts of $6-7$ is a mystery, and self-interacting dark matter could have a major role to play in this story.

If the hidden and visible sectors are closely connected such that the temperatures remain comparable even at late times, the hidden glueballs will generically over close the Universe. We considered a depletion mechanism into right-handed neutrinos and found that it can efficiently remove hidden gluons before confinement. Self-interaction strengths required to explain the astrophysical puzzles on small scales are obtained for glueballino masses $\gtrsim 1 \mathrm{TeV}$ and confinement scales $\sim \mathrm{MeV}$.

The possibility of strong self-interactions in the dark sector is well motivated by observations of lower-than-expected dark matter densities in the centers of galaxies. A strongly interacting hidden sector naturally realizes this possibility. Even in the simple models explored in this paper, we have discovered new features that must be incorporated into numerical simulations to correctly predict the spatial distribution of dark matter in the central parts of structures from dwarf galaxies to clusters of galaxies.

\section{Acknowledgments}

We are grateful for helpful conversations with David B. Kaplan, Jared Kaplan, Matthew Reece, Ira Rothstein, Yael Shadmi, Jessie Shelton, Sean Tulin, and Hai-Bo Yu. K.B. thanks the UC Irvine Department of Physics and Astronomy for hospitality throughout this work. K.B. is supported in part by U.S. DOE grant No. DE-FG02-92ER40701 and by the Gordon and Betty Moore Foundation through Grant No. 776 to the Caltech Moore Center for Theoretical Cosmology and Physics. J.L.F. and T.M.P.T. are supported in part by U.S. NSF grant No. PHY-1316792. T.M.P.T. is further supported in part by the University of California, Irvine through a Chancellor's Fellowship. M.K. is supported in part by NSF Grants No. PHY-1214648 and No. PHY-1316792. We used the LSODA software from LLNL $[104,105]$ to solve the Schrödinger equation. All other calculations were performed with Mathematica 8.0.

[1] M. Rocha, A. H. Peter, J. S. Bullock, M. Kaplinghat, S. Garrison-Kimmel, et al., "Cosmological Simulations with Self-Interacting Dark Matter I: Constant Density Cores and Substructure," MNRAS 430 (2013) 81-104, arXiv:1208.3025 [astro-ph.C0].

[2] A. H. G. Peter, M. Rocha, J. S. Bullock, and M. Kaplinghat, "Cosmological Simulations with Self-Interacting Dark Matter II: Halo Shapes vs. Observations," MNRAS 430 (Mar., 2013) 105-120, arXiv:1208.3026 [astro-ph.CO].

[3] M. Vogelsberger, J. Zavala, and A. Loeb, "Subhaloes in Self-Interacting Galactic Dark Matter Haloes," MNRAS 423 (2012) 3740, arXiv:1201.5892 [astro-ph.CO].

[4] J. Zavala, M. Vogelsberger, and M. G. Walker, "Constraining Self-Interacting Dark Matter with the Milky Way's dwarf spheroidals," MNRAS 431 (2013) L20-L24, arXiv:1211.6426 [astro-ph.Co]. 
[5] T. Hemmick, D. Elmore, T. Gentile, P. Kubik, S. Olsen, et al., "A Search for Anomalously Heavy Isotopes of Low Z Nuclei," Phys.Rev. D41 (1990) 2074-2080.

[6] P. Verkerk, G. Grynberg, B. Pichard, M. Spiro, S. Zylberajch, et al., "Search for superheavy hydrogen in sea water," Phys.Rev.Lett. 68 (1992) 1116-1119.

[7] T. Yamagata, Y. Takamori, and H. Utsunomiya, "Search for anomalously heavy hydrogen in deep sea water at 4000-m," Phys.Rev. D47 (1993) 1231-1234.

[8] L. Randall and R. Sundrum, "Out of this world supersymmetry breaking," Nucl.Phys. B557 (1999) 79-118, arXiv:hep-th/9810155 [hep-th].

[9] G. F. Giudice, M. A. Luty, H. Murayama, and R. Rattazzi, "Gaugino mass without singlets," JHEP 9812 (1998) 027, arXiv:hep-ph/9810442 [hep-ph].

[10] J. L. Feng and J. Kumar, "The WIMPless Miracle: Dark-Matter Particles without Weak-Scale Masses or Weak Interactions," Phys.Rev.Lett. 101 (2008) 231301, arXiv:0803.4196 [hep-ph].

[11] J. L. Feng, H. Tu, and H.-B. Yu, "Thermal Relics in Hidden Sectors," JCAP 0810 (2008) 043, arXiv:0808.2318 [hep-ph].

[12] J. L. Feng and Y. Shadmi, "WIMPless Dark Matter from Non-Abelian Hidden Sectors with Anomaly-Mediated Supersymmetry Breaking," Phys.Rev. D83 (2011) 095011, arXiv:1102.0282 [hep-ph].

[13] J. L. Feng, V. Rentala, and Z. Surujon, "WIMPless Dark Matter in Anomaly-Mediated Supersymmetry Breaking with Hidden QED," Phys.Rev. D84 (2011) 095033, arXiv:1108.4689 [hep-ph].

[14] J. L. Feng, V. Rentala, and Z. Surujon, "WIMPless Dark Matter from an AMSB Hidden Sector with No New Mass Parameters," Phys.Rev. D85 (2012) 055003, arXiv:1111.4479 [hep-ph].

[15] G. D. Kribs, T. S. Roy, J. Terning, and K. M. Zurek, "Quirky Composite Dark Matter," Phys.Rev. D81 (2010) 095001, arXiv:0909.2034 [hep-ph].

[16] D. S. Alves, S. R. Behbahani, P. Schuster, and J. G. Wacker, "Composite Inelastic Dark Matter," Phys.Lett. B692 (2010) 323-326, arXiv:0903.3945 [hep-ph].

[17] A. Falkowski, J. Juknevich, and J. Shelton, "Dark Matter Through the Neutrino Portal," arXiv:0908.1790 [hep-ph].

[18] M. Lisanti and J. G. Wacker, "Parity Violation in Composite Inelastic Dark Matter Models," Phys.Rev. D82 (2010) 055023, arXiv:0911.4483 [hep-ph].

[19] D. Spier Moreira Alves, S. R. Behbahani, P. Schuster, and J. G. Wacker, "The Cosmology of Composite Inelastic Dark Matter," JHEP 1006 (2010) 113, arXiv:1003.4729 [hep-ph].

[20] K. Kumar, A. Menon, and T. M. Tait, "Magnetic Fluffy Dark Matter," JHEP 1202 (2012) 131, arXiv:1111.2336 [hep-ph].

[21] J. M. Cline, Z. Liu, G. Moore, and W. Xue, "Composite strongly interacting dark matter," arXiv:1312.3325 [hep-ph].

[22] J. Kang, M. A. Luty, and S. Nasri, "The Relic abundance of long-lived heavy colored particles," JHEP 0809 (2008) 086, arXiv:hep-ph/0611322 [hep-ph].

[23] WMAP Collaboration, G. Hinshaw et al., "Nine-Year Wilkinson Microwave Anisotropy Probe (WMAP) Observations: Cosmological Parameter Results," ApJS 208 (2013) 19, arXiv:1212.5226 [astro-ph.CO].

[24] Planck Collaboration, P. Ade et al., "Planck 2013 results. XVI. Cosmological parameters," arXiv:1303.5076 [astro-ph.CO].

[25] B. A. Reid, W. J. Percival, D. J. Eisenstein, L. Verde, D. N. Spergel, et al., "Cosmological Constraints from the Clustering of the Sloan Digital Sky Survey DR7 Luminous Red Galaxies," MNRAS 404 (2010) 60-85, arXiv:0907.1659 [astro-ph.CO].

[26] K. N. Abazajian et al., "The Seventh Data Release of the Sloan Digital Sky Survey," ApJS 182 (2009) 543-558, arXiv:0812.0649 [astro-ph].

[27] J. F. Navarro, C. S. Frenk, and S. D. White, "The Assembly of galaxies in a hierarchically clustering universe," MNRAS 275 (1995) 56-66, arXiv:astro-ph/9408067 [astro-ph].

[28] J. F. Navarro, C. S. Frenk, and S. D. White, "The Structure of cold dark matter halos," ApJ 462 (1996) 563-575, arXiv:astro-ph/9508025 [astro-ph].

[29] J. F. Navarro, C. S. Frenk, and S. D. White, "A Universal density profile from hierarchical clustering," ApJ 490 (1997) 493-508, arXiv: astro-ph/9611107 [astro-ph].

[30] J. S. Bullock, T. S. Kolatt, Y. Sigad, R. S. Somerville, A. V. Kravtsov, et al., "Profiles of dark 
haloes. Evolution, scatter, and environment," MNRAS 321 (2001) 559-575, arXiv:astro-ph/9908159 [astro-ph].

[31] R. H. Wechsler, J. S. Bullock, J. R. Primack, A. V. Kravtsov, and A. Dekel, "Concentrations of dark halos from their assembly histories," ApJ 568 (2002) 52-70, arXiv:astro-ph/0108151 [astro-ph].

[32] R. A. Flores and J. R. Primack, "Observational and theoretical constraints on singular dark matter halos," ApJ 427 (1994) L1-4, arXiv:astro-ph/9402004 [astro-ph].

[33] J. D. Simon, A. D. Bolatto, A. Leroy, L. Blitz, and E. L. Gates, "High-resolution measurements of the halos of four dark matter-dominated galaxies: Deviations from a universal density profile," ApJ 621 (2005) 757-776, arXiv:astro-ph/0412035 [astro-ph].

[34] R. Kuzio de Naray, S. S. McGaugh, and W. de Blok, "Mass Models for Low Surface Brightness Galaxies with High Resolution Optical Velocity Fields," ApJ 676 (2008) 920-943, arXiv:0712.0860 [astro-ph].

[35] A. A. Dutton, C. Conroy, F. C. d. Bosch, L. Simard, T. Mendel, et al., "Dark halo response and the stellar initial mass function in early-type and late-type galaxies," MNRAS 416 (2010) 322-345, arXiv:1012.5859 [astro-ph.CO].

[36] R. K. de Naray and K. Spekkens, "Do Baryons Alter the Halos of Low Surface Brightness Galaxies?," ApJ 741 (2011) L29, arXiv:1109.1288 [astro-ph.CO].

[37] S.-H. Oh, W. de Blok, E. Brinks, F. Walter, and J. Kennicutt, Robert C., "Dark and luminous matter in THINGS dwarf galaxies," AJ 141 (2010) 193, arXiv:1011.0899 [astro-ph.C0].

[38] P. Salucci, M. I. Wilkinson, M. G. Walker, G. F. Gilmore, E. K. Grebel, et al., "Dwarf spheroidal galaxy kinematics and spiral galaxy scaling laws," MNRAS 420 (2011) 2034-2041, arXiv:1111.1165 [astro-ph.CO].

[39] G. Castignani, N. Frusciante, D. Vernieri, and P. Salucci, "The density profiles of Dark Matter halos in Spiral Galaxies," Natural Sci. 4 (2012) 265-270, arXiv:1201.3998 [astro-ph.C0].

[40] M. G. Walker and J. Penarrubia, "A Method for Measuring (Slopes of) the Mass Profiles of Dwarf Spheroidal Galaxies," ApJ 742 (2011) 20, arXiv:1108.2404 [astro-ph.CO].

[41] D. J. Sand, T. Treu, G. Smith, and R. Ellis, "The Dark matter distribution in the central regions of galaxy clusters," ApJ 604 (2004) 88-107, arXiv:astro-ph/0310703 [astro-ph].

[42] D. Sand, T. Treu, R. Ellis, G. Smith, and J.-P. Kneib, "Separating baryons and dark matter in cluster cores: A full 2D lensing and dynamic analysis of Abell 383 and MS2137-23," ApJ 674 (Feb, 2008) 711, arXiv:0710.1069 [astro-ph].

[43] A. Newman, T. Treu, R. Ellis, D. Sand, J. Richard, et al., "The Distribution of Dark Matter Over 3 Decades in Radius in the Lensing Cluster Abell 611," ApJ 706 (2009) 1078-1094, arXiv:0909.3527 [astro-ph.CO].

[44] A. B. Newman, T. Treu, R. S. Ellis, and D. J. Sand, "The Dark Matter Distribution in Abell 383: Evidence for a Shallow Density Cusp from Improved Lensing, Stellar Kinematic and X-ray Data," arXiv:1101.3553 [astro-ph.CO].

[45] D. Coe, K. Umetsu, A. Zitrin, M. Donahue, E. Medezinski, et al., "CLASH: Precise New Constraints on the Mass Profile of Abell 2261," ApJ 757 (2012) 22, arXiv:1201.1616 [astro-ph.CO].

[46] K. Umetsu, E. Medezinski, M. Nonino, J. Merten, A. Zitrin, et al., "CLASH: Mass Distribution in and around MACS J1206.2-0847 from a Full Cluster Lensing Analysis," ApJ 755 (2012) 56, arXiv: 1204.3630 [astro-ph.CO].

[47] W. de Blok, "The Core-Cusp Problem," Adv.Astron. 2010 (2010) 789293, arXiv:0910.3538 [astro-ph.CO].

[48] V. Springel, J. Wang, M. Vogelsberger, A. Ludlow, A. Jenkins, et al., "The Aquarius Project: the subhalos of galactic halos," MNRAS 391 (2008) 1685-1711, arXiv:0809.0898 [astro-ph].

[49] M. Boylan-Kolchin, J. S. Bullock, and M. Kaplinghat, "Too big to fail? The puzzling darkness of massive Milky Way subhaloes," MNRAS 415 (2011) L40, arXiv:1103.0007 [astro-ph.C0].

[50] M. Boylan-Kolchin, J. S. Bullock, and M. Kaplinghat, "The Milky Way's bright satellites as an apparent failure of LCDM," MNRAS 422 (2012) 1203-1218, arXiv:1111.2048 [astro-ph.C0].

[51] D. H. Weinberg, J. S. Bullock, F. Governato, R. K. de Naray, and A. H. G. Peter, "Cold dark matter: controversies on small scales," arXiv:1306.0913 [astro-ph.CO].

[52] S.-H. Oh, C. Brook, F. Governato, E. Brinks, L. Mayer, et al., "The central slope of dark matter cores in dwarf galaxies: Simulations vs. THINGS," AJ 142 (2011) 42, arXiv:1011.2777 [astro-ph.CO]. 
[53] F. Governato, A. Zolotov, A. Pontzen, C. Christensen, S. Oh, et al., "Cuspy No More: How Outflows Affect the Central Dark Matter and Baryon Distribution in Lambda CDM Galaxies," MNRAS 422 (2012) 1231-1240, arXiv:1202.0554 [astro-ph.CO].

[54] A. B. Newman, T. Treu, R. S. Ellis, and D. J. Sand, "The Density Profiles of Massive, Relaxed Galaxy Clusters: II. Separating Luminous and Dark Matter in Cluster Cores," ApJ 765 no. issue, (2013) 25, arXiv:1209.1392 [astro-ph.CO].

[55] S. Tremaine and J. Gunn, "Dynamical Role of Light Neutral Leptons in Cosmology," Phys.Rev.Lett. 42 (1979) 407-410.

[56] J. Bond, G. Efstathiou, and J. Silk, "Massive Neutrinos and the Large Scale Structure of the Universe," Phys.Rev.Lett. 45 (1980) 1980-1984.

[57] K. A. Olive and M. S. Turner, "Cosmological bounds on the masses of stable, right-handed neutrinos," Phys.Rev. D25 (1982) 213.

[58] D. N. Spergel and P. J. Steinhardt, "Observational evidence for selfinteracting cold dark matter," Phys.Rev.Lett. 84 (2000) 3760-3763, arXiv:astro-ph/9909386 [astro-ph].

[59] C. Firmani, E. D'Onghia, V. Avila-Reese, G. Chincarini, and X. Hernandez, "Evidence of self-interacting cold dark matter from galactic to galaxy cluster scales," MNRAS 315 (2000) L29, arXiv:astro-ph/0002376 [astro-ph].

[60] C. Firmani, E. D'Onghia, G. Chincarini, X. Hernandez, and V. Avila-Reese, "Constraints on dark matter physics from dwarf galaxies through galaxy cluster haloes," MNRAS 321 (2001) 713, arXiv:astro-ph/0005001 [astro-ph].

[61] E. Polisensky and M. Ricotti, "Constraints on the Dark Matter Particle Mass from the Number of Milky Way Satellites," Phys.Rev. D83 (2011) 043506, arXiv:1004.1459 [astro-ph.Co].

[62] U. Seljak, A. Makarov, P. McDonald, and H. Trac, "Can sterile neutrinos be the dark matter?," Phys.Rev.Lett. 97 (2006) 191303, arXiv:astro-ph/0602430 [astro-ph].

[63] M. Viel, G. D. Becker, J. S. Bolton, and M. G. Haehnelt, "Warm Dark Matter as a solution to the small scale crisis: new constraints from high redshift Lyman-alpha forest data," Physical Review D88 no. 4, (2013) 043502, arXiv:1306.2314 [astro-ph.C0].

[64] R. K. de Naray, G. D. Martinez, J. S. Bullock, and M. Kaplinghat, "The Case Against Warm or Self-Interacting Dark Matter as Explanations for Cores in Low Surface Brightness Galaxies," ApJ 710 (Feb., 2010) L161-L166, arXiv:0912.3518 [astro-ph.C0].

[65] S. W. Randall, M. Markevitch, D. Clowe, A. H. Gonzalez, and M. Bradac, "Constraints on the Self-Interaction Cross-Section of Dark Matter from Numerical Simulations of the Merging Galaxy Cluster 1E 0657-56," ApJ 679 (2008) 1173-1180, arXiv:0704.0261 [astro-ph].

[66] D. D. Dietrich and F. Sannino, "Conformal window of SU(N) gauge theories with fermions in higher dimensional representations," Phys.Rev. D75 (2007) 085018, arXiv:hep-ph/0611341 [hep-ph].

[67] T. Appelquist, A. Ratnaweera, J. Terning, and L. Wijewardhana, "The Phase structure of an SU(N) gauge theory with N(f) flavors," Phys.Rev. D58 (1998) 105017, arXiv:hep-ph/9806472 [hep-ph].

[68] V. Miransky and K. Yamawaki, "Conformal phase transition in gauge theories," Phys.Rev. D55 (1997) 5051-5066, arXiv:hep-th/9611142 [hep-th].

[69] A. G. Cohen and H. Georgi, "Walking beyond the rainbow," Nucl.Phys. B314 (1989) 7.

[70] T. Appelquist, K. D. Lane, and U. Mahanta, "On the ladder approximation for spontaneous chiral symmetry breaking," Phys.Rev.Lett. 61 (1988) 1553.

[71] E. Poppitz and M. Unsal, "Conformality or confinement (II): One-flavor CFTs and mixed-representation QCD," JHEP 0912 (2009) 011, arXiv:0910.1245 [hep-th].

[72] E. Poppitz and M. Unsal, "Conformality or confinement: (IR)relevance of topological excitations," JHEP 0909 (2009) 050, arXiv:0906.5156 [hep-th].

[73] S. Narison, "Masses, decays and mixings of gluonia in QCD," Nucl.Phys.Proc.Suppl. 64 (1998) 210-219, arXiv:hep-ph/9710281 [hep-ph].

[74] C. J. Morningstar and M. J. Peardon, "The Glueball spectrum from an anisotropic lattice study," Phys.Rev. D60 (1999) 034509, arXiv:hep-lat/9901004 [hep-lat].

[75] A. Manohar and H. Georgi, "Chiral Quarks and the Nonrelativistic Quark Model," Nucl.Phys. B234 (1984) 189.

[76] A. G. Cohen, D. B. Kaplan, and A. E. Nelson, "Counting 4 pis in strongly coupled supersymmetry," Phys.Lett. B412 (1997) 301-308, arXiv:hep-ph/9706275 [hep-ph].

[77] B. Lucini, M. Teper, and U. Wenger, "Properties of the deconfining phase transition in SU(N) gauge 
theories," JHEP 0502 (2005) 033, arXiv:hep-lat/0502003 [hep-lat].

[78] G. R. Farrar, "Phenomenology of light gauginos. 1. Motivation, masses, lifetimes and limits," arXiv:hep-ph/9508291 [hep-ph].

[79] S. Raby, "Gauge mediated SUSY breaking at an intermediate scale," Phys.Rev. D56 (1997) 2852-2860, arXiv:hep-ph/9702299 [hep-ph].

[80] S. Raby and K. Tobe, "The Phenomenology of SUSY models with a gluino LSP," Nucl.Phys. B539 (1999) 3-22, arXiv:hep-ph/9807281 [hep-ph].

[81] M. R. Kauth, J. H. Kuhn, P. Marquard, and M. Steinhauser, "Gluinonia: Energy Levels, Production and Decay," Nucl.Phys. B831 (2010) 285-305, arXiv:0910.2612 [hep-ph].

[82] S. Tulin, H.-B. Yu, and K. M. Zurek, "Beyond Collisionless Dark Matter: Particle Physics Dynamics for Dark Matter Halo Structure," Phys.Rev. D87 (2013) 115007, arXiv:1302.3898 [hep-ph].

[83] S. A. Khrapak, A. V. Ivlev, G. E. Morfill, and S. K. Zhdanov, "Scattering in the attractive yukawa potential in the limit of strong interaction," Phys. Rev. Lett. 90 (Jun, 2003) 225002.

[84] S. Khrapak, A. Ivlev, G. Morfill, S. Zhdanov, and H. Thomas, "Scattering in the attractive yukawa potential: application to the ion-drag force in complex plasmas," Plasma Science, IEEE Transactions on 32 no. 2, (April, 2004) $555-560$.

[85] S. A. Khrapak, A. V. Ivlev, and G. E. Morfill, "Momentum transfer in complex plasmas," Phys. Rev. E 70 (Nov, 2004) 056405.

[86] J. L. Feng, M. Kaplinghat, and H.-B. Yu, "Halo Shape and Relic Density Exclusions of Sommerfeld-Enhanced Dark Matter Explanations of Cosmic Ray Excesses," Phys.Rev.Lett. 104 (2010) 151301, arXiv:0911.0422 [hep-ph].

[87] M. Vogelsberger and J. Zavala, "Direct detection of self-interacting dark matter," MNRAS 430 (2013) 1722-1735, arXiv:1211.1377 [astro-ph.C0].

[88] J. Hisano, S. Matsumoto, M. Nagai, O. Saito, and M. Senami, "Non-perturbative effect on thermal relic abundance of dark matter," Phys.Lett. B646 (2007) 34-38, arXiv:hep-ph/0610249 [hep-ph].

[89] A. Hryczuk, R. Iengo, and P. Ullio, "Relic densities including Sommerfeld enhancements in the MSSM," JHEP 1103 (2011) 069, arXiv:1010.2172 [hep-ph].

[90] LEP2 SUSY Working Group, ALEPH, DELPHI, L3 and OPAL experiments, "Charginos, large m0." LEPSUSYWG/01-03.1.

[91] LEP2 SUSY Working Group, ALEPH, DELPHI, L3 and OPAL experiments, "Charginos, at small DM." LEPSUSYWG/02-04.1.

[92] J. F. Hennawi and J. P. Ostriker, "Observational constraints on the self interacting dark matter scenario and the growth of supermassive black holes," ApJ (2001), arXiv:astro-ph/0108203 [astro-ph].

[93] T. Tanaka and Z. Haiman, "The Assembly of Supermassive Black Holes at High Redshifts," Astrophys.J. 696 (2009) 1798-1822, arXiv:0807.4702 [astro-ph].

[94] D. J. Mortlock, S. J. Warren, B. P. Venemans, M. Patel, P. C. Hewett, et al., "A luminous quasar at a redshift of $\mathrm{z}=7.085, "$ Nature $\mathbf{4 7 4}$ (2011) 616, arXiv:1106.6088 [astro-ph.CO].

[95] J. Zavala, M. Vogelsberger, and M. G. Walker, "Constraining Self-Interacting Dark Matter with the Milky Way's dwarf spheroidals," Monthly Notices of the Royal Astronomical Society: Letters 431 (2013) L20-L24, arXiv:1211.6426 [astro-ph.CO].

[96] K. Gultekin, D. O. Richstone, K. Gebhardt, T. R. Lauer, S. Tremaine, et al., "The M-sigma and M-L Relations in Galactic Bulges and Determinations of their Intrinsic Scatter," Astrophys.J. 698 (2009) 198-221, arXiv:0903.4897 [astro-ph.GA].

[97] A. Beifiori, S. Courteau, E. M. Corsini, and Y. Zhu, "On the correlations between galaxy properties and supermassive black hole mass," MNRAS 419 (Jan., 2012) 2497-2528, arXiv:1109.6265 [astro-ph.CO].

[98] K. Abazajian, M. Acero, S. Agarwalla, A. Aguilar-Arevalo, C. Albright, et al., "Light Sterile Neutrinos: A White Paper," arXiv:1204.5379 [hep-ph].

[99] D. E. Kaplan, G. Z. Krnjaic, K. R. Rehermann, and C. M. Wells, "Atomic Dark Matter," JCAP 1005 (2010) 021, arXiv:0909.0753 [hep-ph].

[100] F.-Y. Cyr-Racine and K. Sigurdson, "The Cosmology of Atomic Dark Matter," Phys.Rev. D87 (2013) 103515, arXiv:1209.5752 [astro-ph.CO].

[101] J. M. Cline, Z. Liu, G. Moore, and W. Xue, "Scattering properties of dark atoms and molecules," arXiv:1311.6468 [hep-ph]. 
[102] F. Kahlhoefer, K. Schmidt-Hoberg, M. T. Frandsen, and S. Sarkar, "Colliding clusters and dark matter self-interactions," MNRAS 437 (2014) 2865-2881, arXiv:1308.3419 [astro-ph.CO].

[103] J. Fan, A. Katz, L. Randall, and M. Reece, "Double-Disk Dark Matter," Phys.Dark Univ. 2 (2013) 139-156, arXiv:1303.1521 [astro-ph.CO].

[104] A. Hindmarsh, "ODEPACK, A Systematized Collection of ODE Solvers," vol. 1 of IMACS Transactions on Scientific Computation, pp. 55-64. North-Holland, Amsterdam, 1983.

[105] K. Radhakrishnan and A. C. Hindmarsh, "Description and Use of LSODE, the Livermore Solver for Ordinary Differential Equations," tech. rep., LLNL, December, 1993. 